\title{
Variational Conditions under the Constant Rank Constraint Qualification
}

\author{
$\mathrm{Shu} \mathrm{Lu}$ \\ Department of Statistics and Operations Research, University of North Carolina at Chapel Hill, 355 Hanes \\ Building, CB\#3260, Chapel Hill, NC 27599-3260 \\ email: shulu@email.unc.edu http://www.unc.edu/ shulu/
}

\begin{abstract}
This paper studies solution properties of a parametric variational condition under the constant rank constraint qualification (CRCQ), and properties of its underlying set. We start by showing that if the CRCQ holds at a point in a fixed set, then there exists a one-to-one correspondence between the collection of nonempty faces of the normal cone to the set at that point and the collection of active index sets at points nearby. We then study the behavior of the Euclidean projector, and prove under the CRCQ that the set of multipliers associated with the Euclidean projection is locally a continuous multifunction. Following that, we apply the degree theory to a localized normal map, to show that the combination of the CRCQ and the so-called strong coherent orientation condition suffices for the parametric variational condition to have a locally unique, continuous solution, which is selected from finitely many $C^{1}$ functions. Applications of this result under additional assumptions extend or recover some earlier results on parametric variational inequalities.
\end{abstract}

Key words: variational condition; constant rank constraint qualification; normal map; sensitivity; normal cone; face; Euclidean projection

MSC2000 Subject Classification: Primary: 90C31; Secondary: 49J40, 49J53, 47J20, 90C33

OR/MS subject classification: Primary: Programming: complementarity; Secondary: Programming: nonlinear: theory

1. Introduction Starting from the work of Janin in [8] which showed that the constant rank constraint qualification (CRCQ) implies Abadie's CQ, researchers have been using the CRCQ in analysis of nonlinear programs and variational conditions [2, 4, 11, 12, 17, 20, 21]. Most of the latter references used the CRCQ in a combination with the well known Mangasarian-Fromovitz constraint qualification (MFCQ). It is known that the MFCQ, when combined with a second-order condition, suffices for a nonlinear program to have a locally unique, continuous solution [10], but does not guarantee solution Lipschitz continuity [24]. Assuming that the CRCQ holds additionally makes it possible to obtain solution properties stronger than continuity, such as Lipschitz continuity, B-differentiability and piecewise smoothness; see details in [17, Chapter 4].

One motivation of this paper comes from the above background. We investigate the exact role the CRCQ plays in variational conditions, by analyzing solution properties of variational conditions under the CRCQ alone, without assuming the MFCQ. The result here also applies to nonlinear programs; see Corollary 4.3 at the end of Section 4. For such an analysis one cannot naively follow the method in [10], where Kojima reformulated a nonlinear program into finding zeros of a function of both the variable and multipliers, and applied the degree theory to that function. Without the MFCQ, the degree of the latter function may not be well defined, due to possible unboundedness of the set of multipliers. More importantly, dropping the MFCQ allows the feasible set to be empty under an arbitrarily small perturbation, which makes it hard to apply existing variational analysis results. Neither is it easy to adapt the earlier works under the MFCQ/CRCQ combination to remove their dependence on the MFCQ, because development of those works was based on results in [10] and on local solvability under small perturbations. The analysis in this paper relies on some recent results in [14], which discussed properties of parametric sets under the CRCQ. One result of [14] is that under the CRCQ the Euclidean projection onto the parametric set is locally unique and jointly continuous with respect to the point to be projected and the parameter that determines the set, and is indeed a continuous selection from finitely many $C^{1}$ functions. This result enables us to reformulate the variational condition using a localized normal map and then apply the degree theory to that map.

Another motivation of this paper comes from the observation that the CRCQ is a generalization of both the linear independence constraint qualification (LICQ) and the case in which all constraints are linear. Sets defined by linear constraints are polyhedral convex. Sets defined by nonlinear constraints satisfying the LICQ are locally diffeomorphic to their tangent cones which are polyhedral convex; see [6, 25, 28]. 
The latter two references dealt with a nondegeneracy condition which specializes to the LICQ for the case of standard nonlinear constraints. In this paper we do not attempt to establish a diffeomorphism result for sets defined by nonlinear constraints under the CRCQ, but we will show that such sets bear properties that are closely related to the face structure of polyhedral convex sets. We will use this result to study the Euclidean projectors onto such sets.

The variational condition considered in this paper is of the form

$$
-f(x, u) \in N_{S(u)}(x)
$$

where $f$ is a $C^{1}$ function from an open set $\bar{X} \times \bar{U}$ in $\mathbb{R}^{n} \times \mathbb{R}^{m}$ to $\mathbb{R}^{n}, S(u)$ is a subset of $\mathbb{R}^{n}$ for each $u \in \bar{U}$ defined by

$$
S(u)=\left\{x \in \bar{X} \mid g_{i}(x, u) \leq 0, \quad i \in I, \quad g_{i}(x, u)=0, \quad i \in J\right\}
$$

and $N_{S(u)}(x)$ is the normal cone to $S(u)$ at $x$. In the above equation $I$ and $J$ are disjoint finite index sets, and $g_{i}$ for each $i \in I \cup J$ is a function from $\bar{X} \times \bar{U}$ to $\mathbb{R}$. Unless explicitly stated otherwise, we assume that each $g_{i}$ is $C^{2}$ on $\bar{X} \times \bar{U}$. Throughout this paper we assume that a point $(\bar{x}, \bar{u})$ belongs to $\bar{X} \times \bar{U}$ and satisfies (1), and conduct analysis in some neighborhood of $(\bar{x}, \bar{u})$.

To introduce the definition of the CRCQ, denote the index set of active constraints for a point $(x, u) \in$ $\bar{X} \times \bar{U}$ by

$$
I(x, u)=\left\{i \in I \cup J: g_{i}(x, u)=0\right\} .
$$

The above definition implies $J \subset I(x, u)$ whenever $x \in S(u)$.

Definition 1.1 The $C R C Q$ holds at a point $(x, u) \in \bar{X} \times \bar{U}$ if there exist neighborhoods $X$ of $x$ in $\bar{X}$ and $U$ of $u$ in $\bar{U}$ such that for each $K \subset I(x, u)$ the family $\left\{\nabla_{x} g_{i}\left(x^{\prime}, u^{\prime}\right), \quad i \in K\right\}$ is of constant rank at $\left(x^{\prime}, u^{\prime}\right)$ varies in $X \times U$, where $\nabla_{x} g_{i}\left(x^{\prime}, u^{\prime}\right)$ denotes the gradient of $g_{i}$ with respect to $x$ at $\left(x^{\prime}, u^{\prime}\right)$.

It can be seen from the above definition that if the CRCQ holds at a point $(x, u)$, then it holds at all points in some neighborhood of it. As was shown in [8], if the CRCQ holds at some point $(x, u)$ satisfying $x \in S(u)$, then the tangent cone to $S(u)$ at $x$ is given by

$$
\begin{gathered}
T_{S(u)}(x)=\left\{v \in \mathbb{R}^{n} \mid\left\langle\nabla_{x} g_{i}(x, u), v\right\rangle \leq 0, i \in I(x, u) \cap I,\right. \\
\left.\left\langle\nabla_{x} g_{i}(x, u), v\right\rangle=0, i \in J\right\} .
\end{gathered}
$$

Accordingly, the normal cone to $S(u)$ at $x$ is given by

$$
N_{S(u)}(x)=\{0\} \cup\left\{\sum_{i \in I(x, u)} \lambda_{i} \nabla_{x} g_{i}(x, u) \mid \lambda_{i} \in \mathbb{R}_{+} \text {for each } i \in I(x, u) \cap I\right\} .
$$

We will use the notation $\operatorname{pos}\left\{a_{1}, \ldots, a_{k}\right\}$ to denote the positive hull of a finite set $\left\{a_{1}, \ldots, a_{k}\right\}$ of vectors, and $\operatorname{span}\left\{a_{1}, \ldots, a_{k}\right\}$ to denote the subspace spanned by them. That is,

$$
\operatorname{pos}\left\{a_{1}, \ldots, a_{k}\right\}=\{0\} \cup\left\{\sum_{i=1}^{k} \lambda_{i} a_{i} \mid \lambda_{i} \in \mathbb{R}_{+}\right\} \text {and } \operatorname{span}\left\{a_{1}, \ldots, a_{k}\right\}=\{0\} \cup\left\{\sum_{i=1}^{k} \lambda_{i} a_{i}\right\} .
$$

These definitions ensure that $\operatorname{pos} \emptyset=\operatorname{span} \emptyset=\{0\}$. We can then rewrite (5) as

$$
N_{S(u)}(x)=\operatorname{pos}\left\{\nabla_{x} g_{i}(x, u), i \in I(x, u) \cap I\right\}+\operatorname{span}\left\{\nabla_{x} g_{i}(x, u), i \in J\right\} .
$$

Our work in this paper consists of three parts, one in each of the three subsequent sections. The objective is to provide some new results on the set $S(u)$ and on the variational condition (1) under the CRCQ assumption.

Section 2 investigates the structure of normal cones to $S(u)$, for a fixed $u \in U$. It is known for a polyhedral convex set $P$ containing a point $x$ that the nonempty faces of $N_{P}(x)$ are precisely the normal cones to $P$ contained in $N_{P}(x)$; see, e.g., [33, Lemma 2.4.2] and [15, Proposition 1]. Consequently, there exists a neighborhood $X$ of $x$ in $P$, such that the collection of nonempty faces of $N_{P}(x)$ is precisely the collection of normal cones to $P$ at points in $X$. Because constraints defining the set $S(u)$ in $(2)$ are possibly nonlinear, one cannot expect the normal cones to $S(u)$ at points near $x \in S(u)$ to be exactly faces of $N_{S(u)}(x)$. However, under the CRCQ there is a relation between nonempty faces of $N_{S(u)}(x)$ and the normal cones to $S(u)$ at points near $x$, and we can establish this relation by using active index sets. 
This is done in Theorem 2.1, which constructs a one-to-one correspondence between the collection of nonempty faces of $N_{S(u)}(x)$ and the collection of active index sets at nearby points. This result provides a way to derive information about local geometry of $S(u)$ around $x$ from the face structure of $N_{S(u)}(x)$.

Section 3 continues to focus on the behavior of $S(u)$ for a fixed $u \in U$, and studies the Euclidean projector onto it. By a result in [14], under the CRCQ the Euclidean projector on $S(u)$ has a singlevalued, continuous localization. Using the latter result and the aforementioned Theorem 2.1, we prove that the set of multipliers associated with the Euclidean projection is locally a continuous multifunction. This supplements a result in [20], which proved the so-called lower semicontinuity of the set of multipliers to a perturbed projection problem. We then give a simple proof of the fact that B-derivatives of the Euclidean projector are certain skewed projections onto the critical cone. The latter result was originally proved in [20] under additional convexity assumption.

Section 4 analyzes solution properties of the parametric variational condition (1) under a combination of the CRCQ and the strong coherent orientation condition (SCOC). The SCOC requires a family of matrices to have the same nonzero determinantal sign [4, 17]. Roughly speaking, the CRCQ induces stable behavior of the set $S(u)$, and the SCOC induces stable behavior of the function $f$ in coordination with $S(u)$. Theorem 4.1 says that a combination of these two conditions suffices for (1) to have a locally unique, continuous solution as $u$ varies in a set $U_{2}^{\prime}$ containing $\bar{u}$, and that the solution is selected from finitely many $C^{1}$ functions. The set $U_{2}^{\prime}$ is a neighborhood of $\bar{u}$ in $\left\{u \in \bar{U} \mid S(u) \cap X_{0} \neq \emptyset\right\}$, with $X_{0}$ being some neighborhood of $\bar{x}$ in $\mathbb{R}^{n}$. With no further assumptions one cannot expect $U_{2}^{\prime}$ to be convex or open. The proof of Theorem 4.1 is based on results in [14] and in the preceding section. It also uses some earlier results and techniques, including the coherent orientation condition on normal maps [22, 27, 34], formulas on the B-derivative and some matrix manipulation techniques $[4,17,20]$, the relation between degrees of a function and its B-derivative [19], and the technique used in [10] to prove solution uniqueness. It integrates these results and techniques in a relatively short proof, in which the very basic Lemma 4.1 plays a key role.

Applications of Theorem 4.1 under additional assumptions extend or recover some earlier analytical results on (1). By assuming the MFCQ to hold additionally, we obtain Corollary 4.1, which says that the solution to (1) is a $P C^{1}$ function on a neighborhood of $\bar{u}$ in $\mathbb{R}^{m}$. This is closely related to [17, Theorem 4.2.16] and [4, Theorem 5.4.12]. Results in the latter two references were obtained under additional convexity assumption, and based on results in [10] and on certain implicit function theorems for $P C^{1}$ functions. By assuming that the function $g$ is affine we obtain Corollary 4.2, which says that the solution to (1) is Lipschitz continuous on a convex neighborhood of $\bar{u}$ in the set $U_{2}^{\prime}$. This partially recovers a result in [16]. For comprehensive accounts on perturbational analysis of variational conditions and nonlinear programs, see books [1, 3, 4, 9] and the review paper [29] and references herein.

Except where we explicitly state otherwise, we use $\|\cdot\|$ to denote the Euclidean norm and $B$ to denote the unit open ball around the origin, and all projectors and balls will be Euclidean. We use the notation $g_{K}$ to denote the function consisting of $g_{i}$ for indices $i$ in a set $K \subset I \cup J,|K|$ to denote the cardinality of $K, \operatorname{sgn} x$ to denote the sign of a real number $x$ : $\operatorname{sgn} x=1($ or $0,-1)$ if $x>0($ or $=0,<0)$. We use $\nabla_{x} g_{K}(x, u)$ to denote the Jacobian matrix of $g_{K}$ at $(x, u)$ with respect to $x$.

2. Active index sets v.s. faces of the normal cone In this section we study structure of the set $S(u)$ for a fixed $u \in U$. The main result of this section is Theorem 2.1, which assumes that the CRCQ holds at some $(x, u) \in \bar{X} \times \bar{U}$ and gives a one-to-one correspondence between nonempty faces of $N_{S(u)}(x)$ and active index sets at points in $S(u)$ near $x$. By definition, a face of a convex set $C$ in $\mathbb{R}^{n}$ is a convex subset $F$ of $C$ such that if $x_{1}$ and $x_{2}$ belong to $C$ and $\lambda x_{1}+(1-\lambda) x_{2} \in F$ for some $\lambda \in(0,1)$, then $x_{1}$ and $x_{2}$ belong to $F$ as well [31]. Throughout this section, we only need to assume the functions $g_{i}$ for each $i \in I \cup J$ to be $C^{1}$ on $\bar{X} \times \bar{U}$.

The proof of Theorem 2.1 will use the following lemma, which comes from [14, Corollary 1].

Lemma 2.1 Assume that the CRCQ holds at a point $(x, u) \in \bar{X} \times \bar{U}$. Let $K$ be a subset of $I(x, u)$ such that $\left\{\nabla_{x} g_{i}(x, u), i \in K\right\}$ is linearly independent. Suppose that $j \in I(x, u) \backslash K$ satisfies

$$
\nabla_{x} g_{j}(x, u)=\sum_{i \in K} \lambda_{i} \nabla_{x} g_{i}(x, u)
$$

for some $\lambda \in \mathbb{R}^{|K|}$. Then there exist neighborhoods $X^{\prime}$ of $x$ in $\bar{X}, U^{\prime}$ of $u$ in $\bar{U}$ and $Y$ of $g_{K}(x, u)$ in 
$\mathbb{R}^{|K|}$, with $Y$ being convex, and a $C^{1}$ function $\phi: Y \times U^{\prime} \rightarrow \mathbb{R}$, such that $g_{K}\left(x^{\prime}, u^{\prime}\right) \in Y$ and $g_{j}\left(x^{\prime}, u^{\prime}\right)=$ $\phi\left(g_{K}\left(x^{\prime}, u^{\prime}\right), u^{\prime}\right)$ for each $\left(x^{\prime}, u^{\prime}\right) \in X^{\prime} \times U^{\prime}$. Moreover, for each $i \in K$ and each $\left(y, u^{\prime}\right) \in Y \times U^{\prime}$,

$$
\operatorname{sgn} \frac{\partial}{\partial y_{i}} \phi\left(y, u^{\prime}\right)=\operatorname{sgn} \lambda_{i} .
$$

Lemma 2.1 includes the special case in which $K=\emptyset$ and $\nabla_{x} g_{j}(x, u)=0$. For such a case, the set $Y$ becomes $\{0\}$, and this lemma says that $g_{j}$ depends only on $u^{\prime}$ in $X^{\prime} \times U^{\prime}$. In using this lemma to prove Theorem 2.1 we will only need to deal with a fixed $u$, and will suppress the argument $u^{\prime}$ in the function $\phi$.

THEOREM 2.1 Assume that the CRCQ holds at a point $(x, u) \in \bar{X} \times \bar{U}$ with $x \in S(u)$. Then there exists a neighborhood $X$ of $x$ in $\bar{X}$ such that the following hold.

(a) For each $x^{\prime} \in S(u) \cap X$, the active index set $I\left(x^{\prime}, u\right)$ is a subset of $I(x, u)$ and the CRCQ holds at $\left(x^{\prime}, u\right)$, with $\operatorname{pos}\left\{\nabla_{x} g_{i}(x, u), i \in I\left(x^{\prime}, u\right) \cap I\right\}+\operatorname{span}\left\{\nabla_{x} g_{i}(x, u), i \in J\right\}$ being a nonempty face of $N_{S(u)}(x)$.

(b) For each index set $I^{\prime} \subset I(x, u)$ and each $x^{\prime}$ in $S(u) \cap X$,

$$
\begin{gathered}
\operatorname{pos}\left\{\nabla_{x} g_{i}(x, u), i \in I^{\prime} \cap I\right\}+\operatorname{span}\left\{\nabla_{x} g_{i}(x, u), i \in J\right\} \\
\subset \operatorname{pos}\left\{\nabla_{x} g_{i}(x, u), i \in I\left(x^{\prime}, u\right) \cap I\right\}+\operatorname{span}\left\{\nabla_{x} g_{i}(x, u), i \in J\right\}
\end{gathered}
$$

if and only if $I^{\prime} \subset I\left(x^{\prime}, u\right)$.

(c) For each nonempty face $N$ of $N_{S(u)}(x)$, there exists some $x^{\prime} \in S(u) \cap X$ such that $\operatorname{pos}\left\{\nabla_{x} g_{i}(x, u), i \in I\left(x^{\prime}, u\right) \cap I\right\}+\operatorname{span}\left\{\nabla_{x} g_{i}(x, u), i \in J\right\}=N$.

Proof. To begin with, choose a neighborhood $X$ of $x$ in $\bar{X}$ such that (i) each $x^{\prime} \in X$ satisfies that $I\left(x^{\prime}, u\right) \subset I(x, u)$ and that the CRCQ holds at $\left(x^{\prime}, u\right)$; (ii) $X$ is a subset of the neighborhood $X^{\prime}$ determined in Lemma 2.1 for each pair $(K, j)$ that satisfies the assumptions in Lemma 2.1.

Proof of part (a). Choose $x^{\prime} \in S(u) \cap X$. It suffices to prove that the set

$$
F:=\operatorname{pos}\left\{\nabla_{x} g_{i}(x, u), i \in I\left(x^{\prime}, u\right) \cap I\right\}+\operatorname{span}\left\{\nabla_{x} g_{i}(x, u), i \in J\right\}
$$

is a face of

$$
N_{S(u)}(x)=\operatorname{pos}\left\{\nabla_{x} g_{i}(x, u), i \in I(x, u) \cap I\right\}+\operatorname{span}\left\{\nabla_{x} g_{i}(x, u), i \in J\right\} .
$$

Suppose for the purpose of contradiction that $F$ is not a face of $N_{S(u)}(x)$; then by the definition of faces there exist vectors $a, b, c$, and a scalar $\lambda \in(0,1)$ such that

$$
\sum_{i \in I\left(x^{\prime}, u\right)} a_{i} \nabla_{x} g_{i}(x, u)=\lambda \sum_{i \in I(x, u)} b_{i} \nabla_{x} g_{i}(x, u)+(1-\lambda) \sum_{i \in I(x, u)} c_{i} \nabla_{x} g_{i}(x, u)
$$

with $a_{i} \geq 0$ for each $i \in I\left(x^{\prime}, u\right) \cap I, b_{i} \geq 0$ and $c_{i} \geq 0$ for each $i \in I(x, u) \cap I$, and $\sum_{i \in I(x, u)} b_{i} \nabla_{x} g_{i}(x, u) \notin$ $F$. Because $F$ is a convex cone, we have

$$
\sum_{i \in I(x, u) \backslash I\left(x^{\prime}, u\right)} b_{i} \nabla_{x} g_{i}(x, u) \notin F .
$$

Rearrange (10) to get

$$
0=\sum_{i \in I\left(x^{\prime}, u\right)}\left[-a_{i}+\lambda b_{i}+(1-\lambda) c_{i}\right] \nabla_{x} g_{i}(x, u)+\sum_{i \in I(x, u) \backslash I\left(x^{\prime}, u\right)}\left[\lambda b_{i}+(1-\lambda) c_{i}\right] \nabla_{x} g_{i}(x, u) .
$$

Write $\theta_{i}=-a_{i}+\lambda b_{i}+(1-\lambda) c_{i}$ for each $i \in I\left(x^{\prime}, u\right)$ and $\tau_{i}=\lambda b_{i}+(1-\lambda) c_{i}$ for each $i \in I(x, u) \backslash I\left(x^{\prime}, u\right)$. The scalars $\tau_{i}$ 's are all nonnegative. Moreover, it follows from (11) that $b_{j}>0$ for some $j \in I(x, u) \backslash I\left(x^{\prime}, u\right)$; accordingly, we have $\tau_{j}>0$. Rewrite (12) as

$$
-\tau_{j} \nabla_{x} g_{j}(x, u)=\sum_{i \in I\left(x^{\prime}, u\right)} \theta_{i} \nabla_{x} g_{i}(x, u)+\sum_{i \in I(x, u) \backslash I\left(x^{\prime}, u\right), i \neq j} \tau_{i} \nabla_{x} g_{i}(x, u) .
$$


By Carathéodory's theorem, there exist subsets $I_{1}$ of $I\left(x^{\prime}, u\right)$ and $I_{2}$ of $I(x, u) \backslash\left(I\left(x^{\prime}, u\right) \cup\{j\}\right)$, with $\left\{\nabla_{x} g_{i}(x, u), i \in I_{1} \cup I_{2}\right\}$ being linearly independent, such that

$$
-\tau_{j} \nabla_{x} g_{j}(x, u)=\sum_{i \in I_{1} \cup I_{2}} \rho_{i} \nabla_{x} g_{i}(x, u),
$$

for some $\rho \in \mathbb{R}^{\left|I_{1}\right|+\left|I_{2}\right|}$ with $\rho_{i}>0$ for each $i \in I_{2}$.

Now let $K:=I_{1} \cup I_{2}$. The way we chose $X$ ensures the existence of a convex neighborhood $Y$ of $g_{K}(x, u)$ in $\mathbb{R}^{|K|}$ and a $C^{1}$ function $\phi: Y \rightarrow \mathbb{R}$ such that $g_{K}\left(x^{\prime \prime}, u\right) \in Y$ and $g_{j}\left(x^{\prime \prime}, u\right)=\phi\left(g_{K}\left(x^{\prime \prime}, u\right)\right)$ for each $x^{\prime \prime} \in X$, and

$$
\frac{\partial}{\partial y_{i}} \phi(y)<0
$$

for each $i \in I_{2}$ and each $y \in Y$. The facts $j \in I(x, u)$ and $K \subset I(x, u)$ imply that $g_{j}(x, u)=0$ and $g_{K}(x, u)=0$. We have

$g_{j}\left(x^{\prime}, u\right)=g_{j}\left(x^{\prime}, u\right)-g_{j}(x, u)=\phi\left(g_{K}\left(x^{\prime}, u\right)\right)-\phi\left(g_{K}(x, u)\right)=\phi\left(g_{K}\left(x^{\prime}, u\right)\right)-\phi(0)=\sum_{i \in K} \frac{\partial}{\partial y_{i}} \phi(y) g_{i}\left(x^{\prime}, u\right)$

for some $y$ lying on the line segment between 0 and $g_{K}\left(x^{\prime}, u\right)$, where the last equality follows from the mean value theorem. The way we defined $I_{1}$ and $I_{2}$ implies that $g_{i}\left(x^{\prime}, u\right)=0$ for each $i \in I_{1}$ and $g_{i}\left(x^{\prime}, u\right)<0$ for each $i \in I_{2}$, and the convexity of $Y$ implies that $y \in Y$ and therefore $\frac{\partial}{\partial y_{i}} \phi(y)<0$ for each $i \in I_{2}$. Consequently, we either have $g_{j}\left(x^{\prime}, u\right)>0$ (if $I_{2} \neq \emptyset$ ), or $g_{j}\left(x^{\prime}, u\right)=0$ (if $I_{2}=\emptyset$ ). On the other hand, because $x^{\prime} \in S(u)$ and $j \notin I\left(x^{\prime}, u\right)$, we must have $g_{j}\left(x^{\prime}, u\right)<0$, a contradiction.

Proof of part (b). The "if" direction is trivial. For the "only if" direction, suppose for the purpose of contradiction that there exist $x^{\prime} \in S(u) \cap X$ and $I^{\prime} \subset I(x, u)$ satisfying (9), with $I^{\prime} \backslash I\left(x^{\prime}, u\right) \neq \emptyset$.

Let $j$ belong to $I^{\prime} \backslash I\left(x^{\prime}, u\right)$. Because $J \subset I\left(x^{\prime}, u\right), j$ must belong to $I^{\prime} \cap I$. The inclusion (9) implies that $\nabla_{x} g_{j}(x, u)$ belongs to the set on the right side of it. By an application of Carathéodory's theorem, there exists a subset $K$ of $I\left(x^{\prime}, u\right)$, with $\left\{\nabla_{x} g_{i}(x, u), i \in K\right\}$ being linearly independent, such that

$$
\nabla_{x} g_{j}(x, u)=\sum_{i \in K} \rho_{i} \nabla_{x} g_{i}(x, u)
$$

for some $\rho \in \mathbb{R}^{|K|}$. The way we chose $X$ ensures the existence of a convex neighborhood $Y$ of $g_{K}(x, u)$ in $\mathbb{R}^{|K|}$ and a $C^{1}$ function $\phi: Y \rightarrow \mathbb{R}$ such that $g_{K}\left(x^{\prime \prime}, u\right) \in Y$ and $g_{j}\left(x^{\prime \prime}, u\right)=\phi\left(g_{K}\left(x^{\prime \prime}, u\right)\right)$ for each $x^{\prime \prime} \in X$. In particular,

$$
g_{j}\left(x^{\prime}, u\right)=\phi\left(g_{K}\left(x^{\prime}, u\right)\right)=\phi(0)=\phi\left(g_{K}(x, u)\right)=g_{j}(x, u)=0
$$

where $g_{K}\left(x^{\prime}\right)=g_{K}(x)=0$ because $K \subset I\left(x^{\prime}, u\right) \subset I(x, u)$. This contradicts with the fact that $j \notin$ $I\left(x^{\prime}, u\right)$.

Proof of part (c). Let $N$ be a nonempty face of $N_{S(u)}(x)$. Because $N_{S(u)}(x)$ is a polyhedral convex cone, $N$ is of the form

$$
N=\left\{w \in N_{S(u)}(x) \mid\langle v, w\rangle=0\right\}
$$

for some vector $v$ in the polar of $N_{S(u)}(x)$. Let $K=\left\{i \in I(x, u) \mid\left\langle v, \nabla_{x} g_{i}(x, u)\right\rangle=0\right\}$; note that $J \subset K$. We can then rewrite $N$ as

$$
N=\operatorname{pos}\left\{\nabla_{x} g_{i}(x, u), i \in K \cap I\right\}+\operatorname{span}\left\{\nabla_{x} g_{i}(x, u), i \in J\right\} .
$$

Let $K_{1}$ be a maximal subset of $K$ such that $\left\{\nabla_{x} g_{i}(x, u): i \in K_{1}\right\}$ is linearly independent. Write $K_{2}=K \backslash K_{1}$. The way we chose $X$ ensures the existence of a neighborhood $Y$ of $g_{K_{1}}(x, u)$ in $\mathbb{R}^{\left|K_{1}\right|}$ and a $C^{1}$ function $\Phi: Y \rightarrow \mathbb{R}^{\left|K_{2}\right|}$ such that $g_{K_{1}}\left(x^{\prime}, u\right) \in Y$ for each $x^{\prime} \in X$, with

$$
g_{K_{2}}\left(x^{\prime}, u\right)=\Phi\left(g_{K_{1}}\left(x^{\prime}, u\right)\right) .
$$

Because $\left\{\nabla_{x} g_{i}(x, u): i \in K_{1}\right\}$ is linearly independent, we can apply the classical implicit function theorem to obtain a $C^{1}$ arc $t \rightarrow x(t)$ from an interval $(-\bar{t}, \bar{t})$ to $X$ such that (i) $x(0)=x$, (ii) $x^{\prime}(0)=v$, (iii) $g_{K_{1}}(x(t), u)=0$. To obtain such an arc, we may, for example, partition $x=\left(x_{1}, x_{2}\right)$ such that $\nabla_{x_{1}} g_{K_{1}}(x, u)$ is nonsingular and partition $v=\left(v_{1}, v_{2}\right)$ accordingly, let $x_{2}(t)=x_{2}+v_{2} t$, and then let $x_{1}(t)$ 
be the locally unique solution of $g_{K_{1}}\left(x_{1}(t), x_{2}(t), u\right)=0$. The $\operatorname{arc} x(t)$ satisfies $g_{K}(x(t), u)=0$ for each $t \in(-\bar{t}, \bar{t})$, because

$$
g_{K_{2}}(x(t), u)=\Phi\left(g_{K_{1}}(x(t), u)\right)=\Phi(0)=0 .
$$

Because $\left\langle v, \nabla_{x} g_{i}(x, u)\right\rangle<0$ for each $i \in I(x, u) \backslash K$ and $g_{i}(x, u)<0$ for each $i \in I \backslash I(x, u)$, we can guarantee that $g_{i}(x(t), u)<0$ for each $i \in I \backslash K$ and each $t \in(0, \bar{t})$ by reducing $\bar{t}$ further if necessary. As a result, for each $t \in(0, \bar{t})$ the point $x(t)$ belongs to $S(u) \cap X$, with $I(x(t), u)=K$. This together with (14) proves part (3).

Part (a) of Theorem 2.1 shows that one can map $\left\{I\left(x^{\prime}, u\right), x^{\prime} \in S(u) \cap X\right\}$ to the collection of nonempty faces of $N_{S(u)}(x)$ through the operation

$$
I\left(x^{\prime}, u\right) \rightarrow \operatorname{pos}\left\{\nabla_{x} g_{i}(x, u), i \in I\left(x^{\prime}, u\right) \cap I\right\}+\operatorname{span}\left\{\nabla_{x} g_{i}(x, u), i \in J\right\} .
$$

Parts (b) and (c) of the theorem ensure such a map to be injective and surjective, respectively. In view of Lemma 3.3 of this paper, Theorem 2.1 shows that faces of $N_{S(u)}(x)$ are limits of normal cones to $S(u)$ at points near $x$.

The CRCQ assumption is essential in Theorem 2.1. The following are two examples in which the CRCQ fails. In the first example, part (b) of Theorem 2.1 fails. In the second example, parts (a) and (c) of that theorem fail. For notational simplicity we suppress $u$ in the examples.

EXAmple 2.1 Let $S$ be the subset of $\mathbb{R}^{2}$ defined by two constraints $g_{1}\left(x_{1}, x_{2}\right)=x_{1}^{2}-x_{2} \leq 0$ and $g_{2}\left(x_{1}, x_{2}\right)=-x_{2} \leq 0$. The MFCQ holds at the origin of $\mathbb{R}^{2}$ but the CRCQ fails there. The normal cone $N_{S}(0)$ in this case is the vertical half-line pointing downward through the origin, and it has two nonempty faces. On the other hand, for any neighborhood $X$ of the origin in $\mathbb{R}^{2}$, the collection $\left\{I\left(x^{\prime}\right), x^{\prime} \in S \cap X\right\}$ has three members, namely $\{1,2\},\{1\}$, and $\emptyset$. It is therefore impossible to establish a one-to-one map between the collection of nonempty faces of $N_{S}(0)$ and $\left\{I\left(x^{\prime}\right), x^{\prime} \in S \cap X\right\}$.

EXAmple 2.2 Let $S$ be the subset of $\mathbb{R}^{2}$ defined by three constraints $g_{1}\left(x_{1}, x_{2}\right)=-x_{1}^{2}+x_{2} \leq 0$, $g_{2}\left(x_{1}, x_{2}\right)=-x_{1}^{2}-x_{2} \leq 0$ and $g_{3}\left(x_{1}, x_{2}\right)=-x_{1} \leq 0$. Both the MFCQ and the CRCQ fail at the origin of $\mathbb{R}^{2}$. The normal cone $N_{S}(0)$ in this case is $\mathbb{R}_{-} \times \mathbb{R}$, and it has two nonempty faces. On the other hand, for any neighborhood $X$ of the origin in $\mathbb{R}^{2}$, the collection $\left\{I\left(x^{\prime}\right), x^{\prime} \in S \cap X\right\}$ is $\{\{1,2,3\},\{1\},\{2\}, \emptyset\}$. The latter three active index sets do not correspond to any nonempty face of $N_{S}(0)$. The set $\{0\} \times \mathbb{R}$, which is a nonempty face of $N_{S}(0)$, does not correspond to any active index set.

3. The Euclidean projector In this section we study the Euclidean projector onto the set $S(u)$ for a fixed $u \in U$. Before doing so, we need to introduce the concepts of $P C^{1}$ functions and B-differentiability. Let $G$ be a continuous function from an open set $O$ in $\mathbb{R}^{n}$ to $\mathbb{R}^{m}$. We say $G$ is $P C^{1}$, if for each $x \in O$ there exist an neighborhood $N$ of $x$ in $O$ and a finite collection of $C^{1}$ functions $G_{j}: N \rightarrow \mathbb{R}^{m}, j=1, \cdots, k$, such that the inclusion $G\left(x^{\prime}\right) \in\left\{G_{1}\left(x^{\prime}\right), \cdots, G_{k}\left(x^{\prime}\right)\right\}$ holds for each $x^{\prime} \in N$. The functions $G_{1}, \cdots, G_{k}$ are called selection functions of $G$ around $x$. We say that $G$ is $B$-differentiable at a point $x \in O$ if it is Lipschitz continuous in a neighborhood of $x$ and there is a positively homogeneous function $d G(x): \mathbb{R}^{n} \rightarrow \mathbb{R}^{m}$ having the property that $G(x+h)=G(x)+d G(x)(h)+o(h)$. We call $d G(x)(h)$ the B-derivative of $G$ at $x$ for the direction $h$. It was shown in [33, Proposition 4.1.3] that a $P C^{1}$ function on an open set is B-differentiable at each point in that set.

We also need the concept of localizations of multifunctions. Let $G$ be a multifunction from a topological space $P$ to another topological space $T$, and let $\left(p_{0}, t_{0}\right) \in \operatorname{gph} G$. Let $P_{0}$ and $T_{0}$ be neighborhoods of $p_{0}$ in $P$ and $t_{0}$ in $T$ respectively, and let $G_{0}$ be a multifunction from $P_{0}$ to $T_{0}$. We say that $G_{0}$ is a localization to $P_{0} \times T_{0}$ of $G$, if gph $G_{0}=\operatorname{gph} G \cap\left(P_{0} \times T_{0}\right)$.

The development of this section is based on a result in [14], which says that the CRCQ implies the Euclidean projector onto $S(u)$ to have a single-valued, continuous localization, which is a selection from finitely many $C^{1}$ functions. We will give a precise statement of this result in Lemma 3.1. Before presenting that lemma, we need to define a collection of functions, which will serve as selection functions for the aforementioned localization.

Recall that we assumed from the beginning of this paper that there exists a given point $(\bar{x}, \bar{u})$ satisfying (1). Let $\mathcal{B}$ denote the family of sets $K \subset I(\bar{x}, \bar{u})$ such that the set $\left\{\nabla_{x} g_{i}(\bar{x}, \bar{u}), i \in K\right\}$ is linearly 
independent. For each $K \in \mathcal{B}$, define the following system of equations where $x \in \mathbb{R}^{n}$ and $\lambda \in \mathbb{R}^{|I|+|J|}$ are variables, and $u \in \mathbb{R}^{m}$ and $z \in \mathbb{R}^{n}$ are parameters:

$$
\begin{array}{ll}
x-z+\sum_{i \in K} \nabla_{x} g_{i}(x, u) \lambda_{i}=0, & \text { for each } i \in K, \\
g_{i}(x, u)=0 & \text { for each } i \in(I \cup J) \backslash K .
\end{array}
$$

This is the first-order necessary conditions of the nonlinear program of minimizing $\frac{1}{2}\|z-x\|^{2}$ subject to the equality constraints $g_{i}(x, u)=0$ for $i \in K$. Let $\bar{z}:=\bar{x}$, and note that $g_{i}(\bar{x}, \bar{u})=0$ for each $i \in K$ because $K \subset I(\bar{x}, \bar{u})$. Hence, $(x, \lambda)=(\bar{x}, 0)$ solves (15) under parameter $(\bar{u}, \bar{z})$. Moreover, the Jacobian matrix of the function on the left hand side of (15) with respect to $(x, \lambda)$ at $(\bar{x}, 0, \bar{u}, \bar{z})$ is

$$
\left[\begin{array}{ccc}
I_{n} & \nabla_{x} g_{K}(\bar{x}, \bar{u})^{T} & 0 \\
\nabla_{x} g_{K}(\bar{x}, \bar{u}) & 0 & 0 \\
0 & 0 & I_{|I|+|J|-|K|}
\end{array}\right],
$$

where $I_{n}$ and $I_{|I|+|J|-|K|}$ denote identity matrices of dimensions $n$ and $|I|+|J|-|K|$ respectively. Because the matrix (16) is nonsingular, it follows from the classical implicit function theorem that there exist neighborhoods $X_{K}$ of $\bar{x}$ in $\mathbb{R}^{n}, U_{K}$ of $\bar{u}$ in $\mathbb{R}^{m}$, and $Z_{K}$ of $\bar{z}$ in $\mathbb{R}^{n}$, and Lipschitz continuous $C^{1}$ functions $x^{K}: U_{K} \times Z_{K} \rightarrow X_{K}$ and $\lambda^{K}: U_{K} \times Z_{K} \rightarrow \mathbb{R}^{|I|+|J|}$ such that for each $(u, z) \in U_{K} \times Z_{K}$, $\left(x^{K}(u, z), \lambda^{K}(u, z)\right)$ is the unique solution to (15) in $X_{K} \times \mathbb{R}^{|I|+|J|}$. We have $x^{K}(\bar{u}, \bar{z})=\bar{x}$ and $\lambda^{K}(\bar{u}, \bar{z})=$ 0 . Moreover, for each $(u, z) \in U_{K} \times Z_{K}$, if we write $(x, \lambda)=\left(x^{K}(u, z), \lambda^{K}(u, z)\right)$, then the Jacobian matrices of the functions $x^{K}(\cdot, \cdot)$ and $\lambda^{K}(\cdot, \cdot)$ at $(u, z)$ with respect to $z$ are given by

$$
\left[\begin{array}{c}
\nabla_{z} x^{K}(u, z) \\
\nabla_{z} \lambda^{K}(u, z)
\end{array}\right]=-\left[\begin{array}{ccc}
I_{n}+\sum_{i \in K} \nabla_{x}^{2} g_{i}(x, u) \lambda_{i} & \nabla_{x} g_{K}(x, u)^{T} & 0 \\
\nabla_{x} g_{K}(x, u) & 0 & 0 \\
0 & 0 & I_{|I|+|J|-|K|}
\end{array}\right]^{-1}\left[\begin{array}{c}
-I_{n} \\
0 \\
0
\end{array}\right] .
$$

The following lemma is extracted from [14, Lemma 5 and Proposition 2]. The notation $\left(I+N_{S(u)}\right)^{-1}$ in part (a) of this lemma means the inverse of $I+N_{S(u)}$; here $I$ denotes the identity map from $\mathbb{R}^{n}$ to $\mathbb{R}^{n}$. Thus, two points $x$ and $z$ in $\mathbb{R}^{n}$ satisfy $x \in\left(I+N_{S(u)}\right)^{-1}(z)$ if and only if $z \in x+N_{S(u)}(x)$. The notation $\Pi_{S(u)}(z)$ denotes the Euclidean projection of $z$ onto $S(u)$, i.e., the set of points in $S(u)$ closet to $z$. The statement of part (a) says that the graphs of the two multifunctions, taking $(u, z)$ to $\left(I+N_{S(u)}\right)^{-1}(z)$ and $\Pi_{S(u)}(z)$ respectively, coincide within $\left(U_{0}^{\prime} \times Z_{0}\right) \times X_{0}$, and that they also coincide with the graph of a single-valued function $\pi: U_{0}^{\prime} \times Z_{0} \rightarrow X_{0}$.

Lemma 3.1 Assume that the CRCQ holds at $(\bar{x}, \bar{u})$. Let $\beta$ be a real number such that $\beta>1$. Then there exist open neighborhoods $X_{0}$ of $\bar{x}$ in $\bar{X}, U_{0}$ of $\bar{u}$ in $\bar{U}$ and $Z_{0}$ of $\bar{z}$ in $\mathbb{R}^{n}$, such that the following properties hold with a set $U_{0}^{\prime}$ defined by

$$
U_{0}^{\prime}=\left\{u \in U_{0} \mid S(u) \cap X_{0} \neq \emptyset\right\} .
$$

(a) The localization to $\left(U_{0}^{\prime} \times Z_{0}\right) \times X_{0}$ of the multifunction taking $(u, z) \in \bar{U} \times \mathbb{R}^{n}$ to $\left(I+N_{S(u)}\right)^{-1}(z) \subset$ $\mathbb{R}^{n}$ is a single-valued function $\pi$ that coincides with the localization to $\left(U_{0}^{\prime} \times Z_{0}\right) \times X_{0}$ of the multifunction taking $(u, z) \in \bar{U} \times \mathbb{R}^{n}$ to the Euclidean projection $\Pi_{S(u)}(z)$.

(b) There exist real numbers $M_{1}>0$ and $M_{2}>0$ such that

$$
\left\|\pi(u, z)-\pi\left(u^{\prime}, z^{\prime}\right)\right\| \leq \beta\left\|z-z^{\prime}\right\|+M_{1}\left\|u-u^{\prime}\right\|+M_{2}\left\|u-u^{\prime}\right\|^{\frac{1}{2}}
$$

for each $(u, z)$ and $\left(u^{\prime}, z^{\prime}\right)$ in $U_{0}^{\prime} \times Z_{0}$.

(c) For each $(u, z) \in U_{0}^{\prime} \times Z_{0}$ there exists $K \in \mathcal{B}$ satisfying $\pi(u, z)=x^{K}(u, z)$ and $\lambda^{K}(u, z) \in$ $\mathbb{R}_{+}^{|I|} \times \mathbb{R}^{|J|}$.

In the rest of this paper we will use sets $X_{0}, U_{0}, Z_{0}$ and $U_{0}^{\prime}$ as determined by the above lemma. For convenience of presentation, we will assume without loss of generality the following conditions, by making those sets smaller if necessary.

A1 $I(x, u) \subset I(\bar{x}, \bar{u})$ for each $(x, u) \in X_{0} \times U_{0},\left\{\nabla_{x} g_{i}(x, u), i \in K\right\}$ is of constant rank on $X_{0} \times U_{0}$ for each $K \subset I(\bar{x}, \bar{u})$, and $X_{0} \subset X_{K}, U_{0} \subset U_{K}$ and $Z_{0} \subset Z_{K}$ for each $K \in \mathcal{B}$. 
A2 $f$ is Lipschitz continuous on $X_{0} \times U_{0}$ with constant $\rho$ in the sense that

$$
\left\|f(x, u)-f\left(x^{\prime}, u^{\prime}\right)\right\| \leq \rho\left(\left\|x-x^{\prime}\right\|+\left\|u-u^{\prime}\right\|\right)
$$

for each $(x, u)$ and $\left(x^{\prime}, u^{\prime}\right)$ in $X_{0} \times U_{0}$.

A3 For each $(u, z) \in U_{0}^{\prime} \times Z_{0}, K \in \mathcal{B}$ and $x=\pi(u, z)$, the matrix $I_{n}+\sum_{i \in I \cup J}\left(\lambda^{K}(u, z)\right)_{i} \nabla_{x}^{2} g_{i}(x, u)$ is positive definite.

Among the above, A1 and A2 are standard, and A3 is a consequence of the facts that $\lambda^{K}(\bar{u}, \bar{z})=0$ and that $\nabla_{x}^{2} g_{i}(\cdot, \cdot)$ and $\pi(\cdot, \cdot)$ are continuous. Note that A1 ensures the CRCQ to hold for each $(x, u) \in X_{0} \times U_{0}^{\prime}$ satisfying $x \in S(u)$.

Let $(u, z) \in U_{0}^{\prime} \times Z_{0}$. According to part (a) of Lemma 3.1, the point $\pi(u, z)$ belongs to $X_{0}$ and satisfies $z \in \pi(u, z)+N_{S(u)}(\pi(u, z))$. Because the CRCQ holds at $(\pi(u, z), u)$, we may express $N_{S(u)}(\pi(u, z))$ in the form (6). We have

$$
z-\pi(u, z) \in N_{S(u)}(\pi(u, z))=\operatorname{pos}\left\{\nabla_{x} g_{i}(\pi(u, z), u), i \in I(\pi(u, z)) \cap I\right\}+\operatorname{span}\left\{\nabla_{x} g_{i}(\pi(u, z), u), i \in J\right\}
$$

so the set of multipliers

$$
\begin{array}{r}
M(u, z):=\left\{\lambda \in \mathbb{R}^{|I|+|J|} \mid z-\pi(u, z)=\sum_{i \in I \cup J} \lambda_{i} \nabla_{x} g_{i}(\pi(u, z), u),\right. \\
\lambda_{i} \geq 0 \text { for each } i \in I(\pi(u, z), u) \cap I, \\
\left.\lambda_{i}=0 \text { for each } i \in I \backslash I(\pi(u, z), u)\right\}
\end{array}
$$

is nonempty. By part (c) of Lemma 3.1, there exists $K \in \mathcal{B}$ such that $\pi(u, z)=x^{K}(u, z)$ and $\lambda^{K}(u, z) \in$ $\mathbb{R}_{+}^{|I|} \times \mathbb{R}^{|J|}$. One can easily verify that such $\lambda^{K}(u, z)$ actually belongs to $M(u, z)$. Define an index set $I^{+}(u, z)$ as

$$
I^{+}(u, z)=\left\{i \in I \mid \lambda_{i}>0 \text { for some } \lambda \in M(u, z)\right\}
$$

Clearly, $I^{+}(u, z)$ is a subset of $I(\pi(u, z), u) \cap I$. Indeed, the set

$$
\operatorname{pos}\left\{\nabla_{x} g_{i}(\pi(u, z), u), i \in I^{+}(u, z)\right\}+\operatorname{span}\left\{\nabla_{x} g_{i}(\pi(u, z), u), i \in J\right\}
$$

is the smallest nonempty face of $N_{S(u)}(\pi(u, z))$ containing $z-\pi(u, z)$, as implied by the following lemma.

LEMmA 3.2 Let $I_{0}$ and $J_{0}$ be disjoint finite index sets, and let $a_{i} \in \mathbb{R}^{n}$ for each $i \in I_{0} \cup J_{0}$. Define a polyhedral convex cone $N=\operatorname{pos}\left\{a_{i}, i \in I_{0}\right\}+\operatorname{span}\left\{a_{i}, i \in J_{0}\right\}$, and let $y \in N$. Let $M$ be the set of multipliers associated with $y$ :

$$
M=\left\{\lambda \in \mathbb{R}_{+}^{\left|I_{0}\right|} \times \mathbb{R}^{\left|J_{0}\right|} \mid y=\sum_{i \in I_{0} \cup J_{0}} \lambda_{i} a_{i}\right\},
$$

and let $I^{+}$be a subset of $I_{0}$ defined by

$$
I^{+}=\left\{i \in I_{0} \mid \lambda_{i}>0 \text { for some } \lambda \in M\right\} .
$$

Then the set $F:=\operatorname{pos}\left\{a_{i}, i \in I^{+}\right\}+\operatorname{span}\left\{a_{i}, i \in J_{0}\right\}$ is the smallest nonempty face of $N$ that contains $y$, with $y \in \operatorname{ri} F$ (the relative interior of $F$ ).

Proof. The definitions of $M$ and $I^{+}$imply the existence of $\bar{\lambda} \in M$ such that $\bar{\lambda}_{i}>0$ for each $i \in I^{+}$ and $\bar{\lambda}_{i}=0$ for each $i \in I \backslash I^{+}$. To see this, for each $i \in I^{+}$choose $\lambda^{i} \in M$ such that $\left(\lambda^{i}\right)_{i}>0$, and let $\bar{\lambda}$ be the average for all of these $\lambda^{i}$. For the special case in which $I^{+}=\emptyset$, we may let $\bar{\lambda}$ be any element of $M$.

With the existence of such a $\bar{\lambda}$, it follows from an application of [31, Corollary 6.6.2] that $y \in \operatorname{ri} F$.

Next we prove that $F$ is a face of $N$. Suppose that $v_{1}$ and $v_{2}$ belong to $N, t \in(0,1)$, and that $v:=t v_{1}+(1-t) v_{2}$ belongs to $F$. We need to show that $v_{1}$ and $v_{2}$ belong to $F$ as well. Suppose for the purpose of contradiction that $v_{1} \notin F$. The fact that $v_{1} \in N$ implies the existence of $\mu^{1} \in \mathbb{R}_{+}^{\left|I_{0}\right|} \times \mathbb{R}^{\left|J_{0}\right|}$ such that $v_{1}=\sum_{i \in I_{0} \cup J_{0}} \mu_{i}^{1} a_{i}$, and the fact that $v_{1} \notin F$ implies that $\mu_{j}^{1}>0$ for some $j \in I_{0} \backslash I^{+}$. Because $v_{2} \in N$, there exists $\mu^{2} \in \mathbb{R}_{+}^{\left|I_{0}\right|} \times \mathbb{R}^{\left|J_{0}\right|}$ such that $v_{2}=\sum_{i \in I_{0} \cup J_{0}} \mu_{i}^{2} a_{i}$. The vector $\mu:=\mu^{1} t+\mu^{2}(1-t)$ belongs to $\mathbb{R}_{+}^{\left|I_{0}\right|} \times \mathbb{R}^{\left|J_{0}\right|}$ and satisfies $v=\sum_{i \in I_{0} \cup J_{0}} \mu_{i} a_{i}$ and $\mu_{j}>0$. 
The fact that $v \in F$ implies the existence of $\hat{\mu} \in \mathbb{R}_{+}^{\left|I_{0}\right|} \times \mathbb{R}^{\left|J_{0}\right|}$ such that $v=\sum_{i \in I_{0} \cup J_{0}} \hat{\mu}_{i} a_{i}$ with $\hat{\mu}_{i}=0$ for each $i \in I_{0} \backslash I^{+}$. Hence,

$$
\sum_{i \in I_{0} \cup J_{0}} \mu_{i} a_{i}-\sum_{i \in I_{0} \cup J_{0}} \hat{\mu}_{i} a_{i}=v-v=0 .
$$

Let $\tau$ be a sufficiently small positive real number such that the vector

$$
\hat{\lambda}:=\bar{\lambda}+\tau \mu-\tau \hat{\mu}
$$

belongs to $\mathbb{R}_{+}^{\left|I_{0}\right|} \times \mathbb{R}^{\left|J_{0}\right|}$. It follows from (22) and the fact $\bar{\lambda} \in M$ that $\hat{\lambda} \in M$. Because $j \in I_{0} \backslash I^{+}$, we have $\bar{\lambda}_{j}=\hat{\mu}_{j}=0$, which implies that $\hat{\lambda}_{j}>0$. This contradicts with the way we defined $I^{+}$, and completes the proof that $F$ is a face of $N$.

The facts that $y \in \operatorname{ri} F$ and that $F$ is a face of $N$ together imply that $F$ is the smallest nonempty face of $N$ containing $y$; see [31, Theorem 18.1].

To proceed from here, we need the following concepts about limits and continuity of a multifunction. See [32] for a comprehensive discussion on this subject. Let $F$ be a multifunction from a subset $O$ of $\mathbb{R}^{l}$ to $\mathbb{R}^{m}$. The outer limit of $F$ at a point $x \in O$, denoted by $\lim \sup _{x^{\prime} \rightarrow x} F\left(x^{\prime}\right)$, is the set that consists of all vectors $y$ having the property that there is a sequence $\left\{x^{k}\right\}$ of points of $O$ converging to $x$, and a sequence $\left\{y^{k}\right\}$ converging to $y$, in which for each $k$ the vector $y^{k}$ belongs to $F\left(x^{k}\right)$. The inner limit of $F$ at $x \in O$, denoted by $\liminf _{x^{\prime} \rightarrow x} F\left(x^{\prime}\right)$, is the set that consists of all vectors $y$ having the property that for each sequence $\left\{x^{k}\right\}$ of points of $O$ converging to $x$, there is a sequence $\left\{y^{k}\right\}$ converging to $y$, in which for each $k$ the vector $y^{k}$ belongs to $F\left(x^{k}\right)$. By their definitions, the outer and inner limits are always closed sets, with the inner limit being a subset of the outer limit. Hence, we have

$$
\limsup _{x^{\prime} \rightarrow x} F\left(x^{\prime}\right)=\liminf _{x^{\prime} \rightarrow x} F\left(x^{\prime}\right)
$$

if and only if the former is a subset of the latter, in which case we write

$$
\lim _{x^{\prime} \rightarrow x} F\left(x^{\prime}\right)=\limsup _{x^{\prime} \rightarrow x} F\left(x^{\prime}\right)=\liminf _{x^{\prime} \rightarrow x} F\left(x^{\prime}\right) .
$$

We say that $F$ is continuous at $x$, if $F(x)=\lim _{x^{\prime} \rightarrow x} F\left(x^{\prime}\right)$. We say that $F$ is continuous on $O$ if it is continuous at each $x \in O$.

We will use the following technical lemma later.

Lemma 3.3 Let $O$ be a subset of $\mathbb{R}^{l}$, and let $A_{i}$ be a continuous function from $O$ to $\mathbb{R}^{m}$ for $i=1, \cdots, n$. Suppose that for each $K \subset\{1, \cdots, n\}$ the family $\left\{A_{i}\left(x^{\prime}\right), i \in K\right\}$ is of constant rank as $x^{\prime}$ varies in $O$. Then for each $x \in O$ and each $K \subset\{1, \cdots, n\}$,

$$
\lim _{x^{\prime} \rightarrow x} \operatorname{pos}\left\{A_{i}\left(x^{\prime}\right), i \in K\right\}=\operatorname{pos}\left\{A_{i}(x), i \in K\right\} .
$$

Proof. Let $x \in O$ and $K \subset\{1, \cdots, n\}$ be given. We need to prove

$$
\limsup _{x^{\prime} \rightarrow x} \operatorname{pos}\left\{A_{i}\left(x^{\prime}\right), i \in K\right\} \subset \operatorname{pos}\left\{A_{i}(x), i \in K\right\} \subset \liminf _{x^{\prime} \rightarrow x} \operatorname{pos}\left\{A_{i}\left(x^{\prime}\right), i \in K\right\} .
$$

For the second inclusion in (24), let $y \in \operatorname{pos}\left\{A_{i}(x), i \in K\right\}$. There exists $\lambda \in \mathbb{R}_{+}^{|K|}$ such that $y=$ $\sum_{i \in K} \lambda_{i} A_{i}(x)$. Let $\left\{x^{k}\right\}$ be an arbitrary sequence converging to $x$, and define $y^{k}:=\sum_{i \in K} \lambda_{i} A_{i}\left(x^{k}\right)$. For each $k$ we have $y^{k} \in \operatorname{pos}\left\{A_{i}\left(x^{k}\right), i \in K\right\}$, and the sequence $\left\{y^{k}\right\}$ converges to $y$. This proves the second inclusion in (24).

The rest of this proof is on the first inclusion in (24). Let $y$ belong to $\limsup _{x^{\prime} \rightarrow x} \operatorname{pos}\left\{A_{i}\left(x^{\prime}\right), i \in K\right\}$; then there exist sequences $x^{k} \rightarrow x$ and $y^{k} \rightarrow y$ such that $y^{k} \in \operatorname{pos}\left\{A_{i}\left(x^{k}\right), i \in K\right\}$. By Carathéodory's theorem, for each $k=1,2, \cdots$ there exists a subset $K_{1}(k)$ of $K$ such that

$$
y^{k} \in \operatorname{pos}\left\{A_{i}\left(x^{k}\right), i \in K_{1}(k)\right\},
$$

with $\left\{A_{i}\left(x^{k}\right), i \in K_{1}(k)\right\}$ being linearly independent. By passing to a subsequence if necessary, we may assume that $K_{1}(k) \equiv K_{1}$, where $K_{1}$ is a fixed subset of $K$. The constant rank assumption implies that $\left\{A_{i}(x), i \in K_{1}\right\}$ is linearly independent. Let $\lambda^{k} \in \mathbb{R}^{\left|K_{1}\right|}$ be the unique solution of

$$
y^{k}=\sum_{i \in K_{1}} \lambda_{i}^{k} A_{i}\left(x^{k}\right)
$$


The fact that $y^{k} \in \operatorname{pos}\left\{A_{i}\left(x^{k}\right), i \in K_{1}\right\}$ implies that $\lambda^{k} \geq 0$, and the linear independence of $\left\{A_{i}(x), i \in\right.$ $\left.K_{1}\right\}$ implies that $\lambda^{k}$ is bounded. By passing to a subsequence, we may assume that $\lambda^{k} \rightarrow \lambda$. The vector $\lambda$ is nonnegative and satisfies

$$
y=\sum_{i \in K_{1}} \lambda_{i} A_{i}(x)
$$

which proves that $y$ belongs to $\operatorname{pos}\left\{A_{i}(x), i \in K\right\}$.

Lemma 3.3 is closely related to [20, Lemma 5] and [4, Proposition 3.2.9]. The latter two results are characterizations of the constant rank assumption, but Lemma 3.3 is not, in the sense that (23) may hold when that assumption fails. To see this, consider the following example in which $l=1, m=n=2$, and functions $A_{1}: \mathbb{R} \rightarrow \mathbb{R}^{2}$ and $A_{2}: \mathbb{R} \rightarrow \mathbb{R}^{2}$ are given by

$$
A_{1}(x)=\left[\begin{array}{l}
1 \\
x
\end{array}\right] \quad \text { and } \quad A_{2}(x)=\left[\begin{array}{c}
1 \\
x^{2}
\end{array}\right] .
$$

Let $O$ be the open interval $(-1,1)$. It is not hard to check that (23) holds for each $x \in O$ and each $K \subset\{1,2\}$, but the constant rank condition fails for $K=\{1,2\}$.

We use Lemmas 3.2 and 3.3 to prove the following

Proposition 3.1 Assume that the $C R C Q$ holds at $(\bar{x}, \bar{u})$. Let sets $X_{0}, U_{0}, U_{0}^{\prime}$ and $Z_{0}$ and the function $\pi: U_{0}^{\prime} \times Z_{0} \rightarrow X_{0}$ be determined by Lemma 3.1 and by conditions A1, A2 and A3 in p.7. Define $M(u, z)$ and $I^{+}(u, z)$ for each $(u, z) \in U_{0}^{\prime} \times Z_{0}$ as in (20) and (21). For each $(u, z) \in U_{0}^{\prime} \times Z_{0}$, there exists a neighborhood $Z$ of $z$ in $Z_{0}$, such that

$$
I^{+}(u, z) \cup J \subset I\left(\pi\left(u, z^{\prime}\right), u\right) \subset I(\pi(u, z), u)
$$

for each $z^{\prime} \in Z$.

Proof. Choose $(u, z) \in U_{0}^{\prime} \times Z_{0}$, and let $x=\pi(u, z)$ and $y=z-x$. We have $x \in X_{0} \cap S(u)$, and by A1 the CRCQ holds at $(x, u)$. Determine a neighborhood $X$ of $x$ in $X_{0}$ by applying Theorem 2.1 to $(x, u)$.

Because $N_{S(u)}(x)$ is a polyhedral convex cone of the form (6), it has finitely many faces. The point $y$ belongs to $N_{S(u)}(x)$, so there is a real number $\epsilon>0$, such that each face of $N_{S(u)}(x)$ contains $y$ if and only if it meets the ball $y+2 \epsilon B$. Because $\pi$ is a continuous function, we may choose a neighborhood $Z_{1}$ of $z$ in $Z_{0}$, such that $\pi\left(z^{\prime}, u\right) \in X$ and $z^{\prime}-\pi\left(z^{\prime}, u\right) \in y+\epsilon B$ for each $z^{\prime} \in Z_{1}$.

On the other hand, because the CRCQ holds at $(x, u)$, we have by Lemma 3.3

$$
\begin{aligned}
& \limsup _{x^{\prime} \rightarrow x}\left(\operatorname{pos}\left\{\nabla_{x} g_{i}\left(x^{\prime}, u\right), i \in K \cap I\right\}+\operatorname{span}\left\{\nabla_{x} g_{i}\left(x^{\prime}, u\right), i \in J\right\}\right) \\
= & \operatorname{pos}\left\{\nabla_{x} g_{i}(x, u), i \in K \cap I\right\}+\operatorname{span}\left\{\nabla_{x} g_{i}(x, u), i \in J\right\}
\end{aligned}
$$

for each $K \subset I(x, u)$. It then follows from [32, Proposition 5.12] that for each $K \subset I(x, u)$ there is a neighborhood $X^{K}$ of $x$ such that

$$
\begin{aligned}
& \left(\operatorname{pos}\left\{\nabla_{x} g_{i}\left(x^{\prime}, u\right), i \in K \cap I\right\}+\operatorname{span}\left\{\nabla_{x} g_{i}\left(x^{\prime}, u\right), i \in J\right\}\right) \cap(y+\epsilon B) \\
\subset & \operatorname{pos}\left\{\nabla_{x} g_{i}(x, u), i \in K \cap I\right\}+\operatorname{span}\left\{\nabla_{x} g_{i}(x, u), i \in J\right\}+\epsilon B
\end{aligned}
$$

for each $x^{\prime} \in X^{K}$. Now choose a neighborhood $Z$ of $z$ in $Z_{1}$, such that $\pi\left(z^{\prime}, u\right) \in X^{K}$ for each $z^{\prime} \in Z$ and $K \subset I(x, u)$. We prove (25) holds for each $z^{\prime} \in Z$.

Choose $z^{\prime} \in Z$. The fact that $Z \subset Z_{1}$ implies that $\pi\left(z^{\prime}, u\right) \in X$, and the way we chose $X$ implies that $I\left(\pi\left(z^{\prime}, u\right), u\right) \subset I(x, u)$. This proves the second inclusion in (25). For the first inclusion, write $x^{\prime}=\pi\left(z^{\prime}, u\right)$. As $J \subset I\left(x^{\prime}, u\right)$ trivially holds, it suffices to prove $I^{+}(u, z) \subset I\left(x^{\prime}, u\right) \cap I$. Note that

$$
z^{\prime}-x^{\prime} \in y+\epsilon B
$$

by the way we chose $Z_{1}$ and that

$$
z^{\prime}-x^{\prime} \in N_{S(u)}\left(x^{\prime}\right)=\operatorname{pos}\left\{\nabla_{x} g_{i}\left(x^{\prime}, u\right), i \in I\left(x^{\prime}, u\right) \cap I\right\}+\operatorname{span}\left\{\nabla_{x} g_{i}\left(x^{\prime}, u\right), i \in J\right\} .
$$

Because $x^{\prime} \in X^{K}$ for the specific choice of $K=I\left(x^{\prime}, u\right)$, it follows from (26) that

$$
z^{\prime}-x^{\prime} \in \operatorname{pos}\left\{\nabla_{x} g_{i}(x, u), i \in I\left(x^{\prime}, u\right) \cap I\right\}+\operatorname{span}\left\{\nabla_{x} g_{i}(x, u), i \in J\right\}+\epsilon B .
$$


This and (27) imply that the ball $y+2 \epsilon B$ meets the set

$$
\operatorname{pos}\left\{\nabla_{x} g_{i}(x, u), i \in I\left(x^{\prime}, u\right) \cap I\right\}+\operatorname{span}\left\{\nabla_{x} g_{i}(x, u), i \in J\right\} .
$$

Due to the fact that $x^{\prime} \in X$ and the way we chose $X$, the set (28) is a face of $N_{S(u)}(x)$, so it contains $y$ by the way we chose $\epsilon$.

On the other hand, it follows from an application of Lemma 3.2 that the set $F:=\operatorname{pos}\left\{\nabla_{x} g_{i}(x, u), i \in\right.$ $\left.I^{+}(u, z)\right\}+\operatorname{span}\left\{\nabla_{x} g_{i}(x, u), i \in J\right\}$ is the smallest nonempty face of $N_{S(u)}(x)$ that contains $y$. Hence, $F$ is a subset of the set (28). By part (b) of Theorem 2.1, we have $I^{+}(u, z) \subset I\left(x^{\prime}, u\right) \cap I$. This proves the first inclusion of (25).

The following theorem says that the set of multipliers $M(u, z)$ is a continuous multifunction with respect to $z$ for each fixed $u \in U_{0}^{\prime}$. It is related to [20, Lemma 6], which says that the set of multipliers to a perturbed projection problem is "lower semicontinuous." The so-called lower semicontinuity in the latter reference is called inner semicontinuity in [32].

Theorem 3.1 Assume the hypotheses and notation in Proposition 3.1. For each $u \in U_{0}^{\prime}, M(u, \cdot)$ is a continuous multifunction on $Z_{0}$.

Proof. Choose $u \in U_{0}^{\prime}, z \in Z_{0}$, and let $x=\pi(u, z)$. Using the definition of $M$ and the continuity of $\pi(\cdot, \cdot)$ and $\nabla_{x} g_{i}(\cdot, \cdot)$, one can easily verify that

$$
\limsup _{z^{\prime} \rightarrow z} M\left(u, z^{\prime}\right) \subset M(u, z) .
$$

It remains to prove

$$
M(u, z) \subset \liminf _{z^{\prime} \rightarrow z} M\left(u, z^{\prime}\right) .
$$

Note that the set $\liminf _{z^{\prime} \rightarrow z} M\left(u, z^{\prime}\right)$ is closed. Because $M(u, z)$ is convex, it suffices to prove its relative interior, ri $M(u, z)$, is a subset of $\liminf _{z^{\prime} \rightarrow z} M\left(u, z^{\prime}\right)$.

Select an arbitrary $\bar{\lambda} \in \operatorname{ri} M(u, z)$ and a sequence $\left\{z^{k}\right\}$ in $Z_{0}$ converging to $z$. We need to show that there exists $\bar{\lambda}^{k} \in M\left(u, z^{k}\right)$ for each $k$ with $\bar{\lambda}^{k}$ converging to $\bar{\lambda}$. The fact that $\bar{\lambda} \in \operatorname{ri} M(u, z)$ implies that $\bar{\lambda}_{i}>0$ for each $i \in I^{+}(u, z)$ and $\bar{\lambda}_{i}=0$ for each $i \in I \backslash I^{+}(u, z)$.

Let $x^{k}=\pi\left(z^{k}, u\right)$ for each $k$. By passing to a subsequence if necessary, we may assume without loss of generality that $I\left(x^{k}, u\right)$ equals a constant index set $\hat{I}$ for all $k$. It follows from Proposition 3.1 that

$$
I^{+}(u, z) \cup J \subset \hat{I} \subset I(x, u) .
$$

Because $z^{k} \in Z_{0}$, we have

$$
z^{k}-x^{k} \in N_{S(u)}\left(x^{k}\right)=\operatorname{pos}\left\{\nabla_{x} g_{i}\left(x^{k}, u\right), i \in \hat{I} \cap I\right\}+\operatorname{span}\left\{\nabla_{x} g_{i}\left(x^{k}, u\right), i \in J\right\} .
$$

Applying Carathéodory's theorem and passing to a subsequence if necessary, we may assume that there exists a subset $K \subset \hat{I}$, such that $\left\{\nabla_{x} g_{i}\left(x^{k}, u\right), i \in K\right\}$ is linearly independent for each $k$, with

$$
z^{k}-x^{k} \in \operatorname{pos}\left\{\nabla_{x} g_{i}\left(x^{k}, u\right), i \in K \cap I\right\}+\operatorname{span}\left\{\nabla_{x} g_{i}\left(x^{k}, u\right), i \in K \cap J\right\} .
$$

By the way we chose $X_{0}$ and $U_{0}^{\prime}$ (see condition A1), the family $\left\{\nabla_{x} g_{i}\left(x^{\prime}, u\right), i \in K\right\}$ is linearly independent for each $x^{\prime} \in X_{0}$ and $u \in U_{0}^{\prime}$, so $K$ belongs to the collection $\mathcal{B}$ defined at the beginning of this section. The inclusion (33) implies the existence of a vector $\lambda^{k} \in \mathbb{R}^{|I|+|J|}$ satisfying

$$
z^{k}-x^{k}=\sum_{i \in I \cup J} \lambda_{i}^{k} \nabla_{x} g_{i}\left(x^{k}, u\right)
$$

with $\lambda_{i}^{k} \geq 0$ for each $i \in K \cap I$ and $\lambda_{i}^{k}=0$ for each $i \in(I \cup J) \backslash K$. We have $\lambda^{k}=\lambda^{K}\left(u\right.$, $\left.z^{k}\right)$, where the function $\lambda^{K}$ is defined right below (16). We also have $\lambda^{k} \in M\left(u, z^{k}\right)$. The sequence $\left\{\lambda^{k}\right\}$ converges to $\lambda:=\lambda^{K}(u, z)$, and it follows from (29) that $\lambda \in M(u, z)$.

Let $\Delta \lambda=\bar{\lambda}-\lambda$. Because both $\bar{\lambda}$ and $\lambda$ belong to $M(u, z)$, we have

$$
\Delta \lambda_{i}=\bar{\lambda}_{i}=\lambda_{i}=0
$$

for each $i \in I \backslash I^{+}(u, z)$ by the definition of $I^{+}(u, z)$, and

$$
\sum_{i \in I^{+}(u, z) \cup J} \Delta \lambda_{i} \nabla_{x} g_{i}(x, u)=\sum_{i \in I \cup J} \Delta \lambda_{i} \nabla_{x} g_{i}(x, u)=\sum_{i \in I \cup J} \bar{\lambda}_{i} \nabla_{x} g_{i}(x, u)-\sum_{i \in I \cup J} \lambda_{i} \nabla_{x} g_{i}(x, u)=0 .
$$


By [20, Lemma 5], there exists a sequence $\left\{\Delta \lambda^{k}\right\}$ converging to $\Delta \lambda$ such that $\Delta \lambda_{i}^{k}=0$ for each $k$ and each $i \in I \backslash I^{+}(u, z)$, and

$$
\sum_{i \in I \cup J} \Delta \lambda_{i}^{k} \nabla_{x} g_{i}\left(x^{k}, u\right)=0
$$

for each $k$.

Define $\bar{\lambda}^{k}=\Delta \lambda^{k}+\lambda^{k}$ for each $k$. Because $\left\{\Delta \lambda^{k}\right\}$ and $\left\{\lambda^{k}\right\}$ converge to $\Delta \lambda$ and $\lambda$ respectively, the sequence $\left\{\bar{\lambda}^{k}\right\}$ converges to $\bar{\lambda}$.

In the following, we select an arbitrary $k$ and show that $\bar{\lambda}^{k}$ belongs to $M\left(u, z^{k}\right)$. Equation (34) and the fact that $\lambda^{k} \in M\left(u, z^{k}\right)$ together imply

$$
\sum_{i \in I \cup J} \bar{\lambda}_{i}^{k} \nabla_{x} g_{i}\left(x^{k}, u\right)=z-x^{k} .
$$

In view of the fact $\hat{I}=I\left(x^{k}, u\right)$, it remains to show that $\bar{\lambda}_{i}^{k} \geq 0$ for each $i \in \hat{I} \cap I$ and $\bar{\lambda}_{i}^{k}=0$ for each $i \in I \backslash \hat{I}$.

Using (31), we may partition $I$ as the union of three disjoint sets, namely $I^{+}(u, z),(I \cap \hat{I}) \backslash I^{+}(u, z)$ and $I \backslash \hat{I}$. The way we chose $\lambda^{k}$ guarantees that $\lambda_{i}^{k} \geq 0$ for each $i \in I$ and $\lambda_{i}^{k}=0$ for each $i \in I \backslash \hat{I}$ (note that $K \subset \hat{I}$ ). The way we chose $\Delta \lambda^{k}$ guarantees that $\Delta \lambda_{i}^{k}=0$ for each $i \in I \backslash I^{+}(u, z)$. It follows that $\bar{\lambda}_{i}^{k} \geq 0$ for each $i \in(I \cap \hat{I}) \backslash I^{+}(u, z)$ and $\bar{\lambda}_{i}^{k}=0$ for each $i \in I \backslash \hat{I}$. It remains to consider each $i \in I^{+}(u, z)$. For each such $i$ the quantity $\bar{\lambda}_{i}$ is strictly positive, and is the limit of the sequence $\left\{\bar{\lambda}_{i}^{k}\right\}$. Thus, by considering a truncated sequence if necessary, we may assume without loss of generality that $\bar{\lambda}_{i}^{k} \geq 0$. This completes the proof of the fact $\bar{\lambda}^{k} \in M\left(u, z^{k}\right)$.

The CRCQ assumption is essential in Theorem 3.1. Consider Example 2.1 again. The set $S$ is closed and convex, so each $z \in \mathbb{R}^{2}$ has a unique Euclidean projection onto $S$. Because the MFCQ holds at each point of $S$, the set of multipliers associated with the Euclidean projection of each $z \in \mathbb{R}^{2}$, denoted by $M(z)$ here, is well defined. Let $t$ be a negative real number. The Euclidean projection of the point $(0, t)$ onto $S$ is the origin of $\mathbb{R}^{2}$, with

$$
M(0, t)=\left\{\lambda \in \mathbb{R}_{+}^{2}: \lambda_{1}+\lambda_{2}=-t\right\} .
$$

The Euclidean projection of each point $(\epsilon, t)$ in $\mathbb{R}^{2}$ with $\epsilon \neq 0$ is not the origin of $\mathbb{R}^{2}$, and $M(\epsilon, t)$ is a singleton. One can easily verity that $M$ is not continuous at $(0, t)$. Hence, if we let $\bar{z}$ be the origin of $\mathbb{R}^{2}$, then it is impossible to find a neighborhood $Z_{0}$ of $\bar{z}$ in $\mathbb{R}^{2}$ such that $M$ is continuous on $Z_{0}$.

According to parts (b) and (c) of Lemma 3.1, for each fixed $u \in U_{0}^{\prime}$ the function $\pi(u, \cdot)$ is $P C^{1}$ on the open set $Z_{0}$, and is therefore B-differentiable on $Z_{0}$. For each $(u, z) \in U_{0}^{\prime} \times Z_{0}$, denote the B-derivative of $\pi(u, \cdot)$ at $z$ for any direction $h \in \mathbb{R}^{n}$ by $d_{z} \pi(u, z)(h)$, and define the critical cone to $S(u)$ associated with $z$ by

$$
K(u, z)=T_{S(u)}(\pi(u, z)) \cap\{z-\pi(u, z)\}^{\perp} .
$$

Because the CRCQ holds at $(\pi(u, z), u), T_{S(u)}(\pi(u, z))$ is a polyhedral convex cone of the form (4). Consequently, $K(u, z)$ is a polyhedral convex cone as well. In Proposition 3.2 below, we use Theorem 3.1 to give a simple proof of the fact that $d_{z} \pi(u, z)(h)$ solves a certain variational inequality over $K(u, z)$. This result was proved in [20, Theorem 8] under the additional assumptions that $J=\emptyset$ and that for each $i$ the function $g_{i}$ is convex with respect to $x$.

The critical cone $K(u, z)$ has an alternative expression. Let $(u, z) \in U_{0}^{\prime} \times Z_{0}$ and write $x=\pi(u, z)$. The definition of $I^{+}(u, z)$ implies the existence of $\lambda \in M(u, z)$ with $\lambda_{i}>0$ for each $i \in I^{+}(u, z)$ and $\lambda_{i}=0$ for each $i \in I \backslash I^{+}(u, z)$. Because $z-x$ is in the polar of $T_{S(u)}(x)$, each $v \in T_{S(u)}(x)$ satisfies $\langle z-x, v\rangle=0$ if and only if $\left\langle\nabla_{x} g_{i}(x, u), v\right\rangle=0$ for each $i \in I^{+}(u, z)$. Hence, we can rewrite (36) as

$$
\begin{aligned}
K(u, z) & =\left\{v \in T_{S(u)}(x) \mid\left\langle\nabla_{x} g_{i}(x, u), v\right\rangle=0, i \in I^{+}(u, z)\right\} \\
& =\left\{v \in \mathbb{R}^{n} \mid \begin{array}{l}
\left\langle\nabla_{x} g_{i}(x, u), v\right\rangle \leq 0, i \in I(x, u) \cap I \\
\left\langle\nabla_{x} g_{i}(x, u), v\right\rangle=0, i \in I^{+}(u, z) \cup J
\end{array}\right\} .
\end{aligned}
$$

Proposition 3.2 Assume the hypotheses and notation in Proposition 3.1. Let $(u, z) \in U_{0}^{\prime} \times Z_{0}, x=$ $\pi(u, z), \lambda \in M(u, z)$, and $h \in \mathbb{R}^{n}$. Write $A:=I_{n}+\sum_{i \in I \cup J} \lambda_{i} \nabla_{x}^{2} g_{i}(x, u)$ and $w:=d_{z} \pi(u, z)(h)$. Then $w \in K(u, z)$ and solves the variational inequality

$$
\langle h-A w, v-w\rangle \leq 0 \text { for each } v \in K(u, z) .
$$


Proof. The fact that $w$ belongs to $K(u, z)$ was originally proved in [7, Proposition 1]; for completeness we give a simple proof below. Let $\left\{\tau^{k}\right\}$ be a sequence of positive scalars converging to zero, and let $z^{k}=z+\tau^{k} h$. Assume without loss of generality that $z^{k} \in Z_{0}$ for each $k$, and write $x^{k}=\pi\left(u, z^{k}\right)$. The fact that $\pi(u, \cdot)$ is B-differentiable at $z$ implies that

$$
\lim _{k \rightarrow \infty}\left(x^{k}-x\right) / \tau^{k}=\lim _{k \rightarrow \infty}\left(\pi\left(u, z+\tau^{k} h\right)-\pi(u, z)\right) / \tau^{k}=d_{z} \pi(u, z)(h)=w .
$$

By passing to a subsequence if necessary, we may assume without loss of generality that the active index set $I\left(x^{k}, u\right)$ equals a constant index set $\hat{I}$ for all $k$. It then follows from Proposition 3.1 that

$$
I^{+}(u, z) \cup J \subset \hat{I} \subset I(x, u) .
$$

Equation (39) implies that $w$ belongs to $T_{S(u)}(x)$, and that

$$
\left\langle\nabla_{x} g_{i}(x, u), w\right\rangle=0 \text { for each } i \in \hat{I}
$$

because $g_{i}\left(x^{k}, u\right)=0$ for each $i \in \hat{I}$ and each $k$. It follows from (37), (40) and (41) that $w \in K(u, z)$.

Next we prove (38). By Theorem 3.1, there exists $\lambda^{k} \in M\left(u, z^{k}\right)$ for each $k$ such that $\lim _{k \rightarrow \infty} \lambda^{k}=\lambda$. We have

$$
\begin{gathered}
\sum_{i \in I \cup J} \lambda_{i}^{k} \nabla_{x} g_{i}\left(x^{k}, u\right)=z^{k}-x^{k}, \\
\sum_{i \in I \cup J} \lambda_{i} \nabla_{x} g_{i}(x, u)=z-x,
\end{gathered}
$$

and

$$
\nabla_{x} g_{i}\left(x^{k}, u\right)=\nabla_{x} g_{i}(x, u)+\nabla_{x}^{2} g_{i}(x, u)\left(x^{k}-x\right)+o\left(\left\|x^{k}-x\right\|\right) .
$$

Substituting the latter expression into (42) and then subtracting (43), and dividing by $\tau^{k}$, we obtain

$$
\sum_{i \in I \cup J}\left(\frac{\lambda_{i}^{k}-\lambda_{i}}{\tau^{k}} \nabla_{x} g_{i}(x, u)+\lambda_{i}^{k} \nabla_{x}^{2} g_{i}(x, u) \frac{x^{k}-x}{\tau^{k}}\right)+\frac{o\left(\left\|x^{k}-x\right\|\right)}{\tau^{k}}=h-\frac{x^{k}-x}{\tau^{k}} .
$$

Taking limits on both sides of the above equation, and simplifying, we have

$$
\lim _{k \rightarrow \infty} \sum_{i \in I \cup J} \frac{\lambda_{i}^{k}-\lambda_{i}}{\tau^{k}} \nabla_{x} g_{i}(x, u)=h-A w .
$$

Using (40), we may partition the set $I \cup J$ as the union of three disjoint sets, namely $I \backslash \hat{I}, I^{+}(u, z) \cup J$ and $\hat{I} \backslash\left(I^{+}(u, z) \cup J\right)$. We treat each of these separately below.

For each $i \in I \backslash \hat{I}$, the facts $I\left(x^{k}, u\right) \equiv \hat{I}$ and $\lambda^{k} \in M\left(u, z^{k}\right)$ imply that $\lambda_{i}^{k}=0$ for each $k$. It follows that $\lambda_{i}=0$ as well.

For each $i \in I^{+}(u, z) \cup J$, the expression of $K(u, z)$ in (37) and the fact that $w \in K(u, z)$ imply that $\left\langle\nabla_{x} g_{i}(x, u), v-w\right\rangle=0$ for each $v \in K(u, z)$.

Finally, the set $\hat{I} \backslash\left(I^{+}(u, z) \cup J\right)$ is a subset of $I$. For each $i$ in it, we have $\lambda_{i}=0$ by the definition of $I^{+}(u, z), \lambda_{i}^{k} \geq 0$ for each $k$ because $\lambda^{k} \in M\left(u, z^{k}\right)$, and by (37), (40) and (41)

$$
\left\langle\nabla_{x} g_{i}(x, u), v-w\right\rangle=\left\langle\nabla_{x} g_{i}(x, u), v\right\rangle-\left\langle\nabla_{x} g_{i}(x, u), w\right\rangle \leq 0
$$

for each $v \in K(u, z)$.

A combination of the above facts yields

$$
\lim _{k \rightarrow \infty} \sum_{i \in I \cup J} \frac{\lambda_{i}^{k}-\lambda_{i}}{\tau^{k}}\left\langle\nabla_{x} g_{i}(x, u), v-w\right\rangle=\lim _{k \rightarrow \infty} \sum_{i \in \hat{I} \backslash\left(I^{+}(u, z) \cup J\right)} \frac{\lambda_{i}^{k}}{\tau^{k}}\left\langle\nabla_{x} g_{i}(x, u), v-w\right\rangle \leq 0
$$

for each $v \in K(u, z)$. This and (45) together imply that (38) holds.

As noted in the remark right below (20), for each $(u, z) \in U_{0}^{\prime} \times Z_{0}$ there exists $K \in \mathcal{B}$ such that $\pi(u, z)=x^{K}(u, z)$ and $\lambda^{K}(u, z) \in M(u, z)$. If we use such $\lambda^{K}(u, z)$ to define the matrix $A$ in Proposition 3.2 , then $A$ is positive definite in view of A3 in p.7. In this case, the variational inequality (38) is equivalent to the strictly convex quadratic program of minimizing $\frac{1}{2} w^{T} A w-h^{T} w$ subject to $w \in K(u, z)$, and has a unique solution. One can replace the objective function of the latter program by $\frac{1}{2}\left(A^{-1} h-\right.$ $w)^{T} A\left(A^{-1} h-w\right)$ without changing its solution. Thus, we denote the unique solution of $(38)$ by $\Pi_{K(u, z)}^{A}(h)$ when $A$ is positive definite, because it is the projection of $A^{-1} h$ on $K(u, z)$ under the socalled $A$-norm $\|w\|_{A}=\left(w^{T} A w\right)^{1 / 2}$. The latter notation comes from [20]. 
4. The variational condition This section analyzes solution properties of the parametric variational condition (1), by applying the degree theory to a localized normal map. Generally, for a closed convex subset $C$ of $\mathbb{R}^{n}$ and a function $G: \mathbb{R}^{n} \rightarrow \mathbb{R}^{n}$, the normal map induced by $G$ on $C$ is a map $G_{C}: \mathbb{R}^{n} \rightarrow \mathbb{R}^{n}$ defined by

$$
G_{C}(z)=G\left(\Pi_{C}(z)\right)+z-\Pi_{C}(z),
$$

where $\Pi_{C}(z)$ is the Euclidean projection of $z$ on $C$; see [27]. Because the set $S(u)$ in this paper is possibly nonconvex, we cannot define normal maps on $S(u)$ as above. However, Lemma 3.1 enables us to define a localized normal map, which was introduced in [30].

Throughout this section, we continue to assume that the CRCQ holds at $(\bar{x}, \bar{u})$, and let sets $X_{0}, U_{0}$, $U_{0}^{\prime}$ and $Z_{0}$ and the function $\pi: U_{0}^{\prime} \times Z_{0} \rightarrow X_{0}$ be determined by Lemma 3.1 and by Conditions A1, A2 and $\mathrm{A} 3$ in p.7.

Now write $\hat{z}:=\bar{x}-f(\bar{x}, \bar{u})$. Without loss of generality, we may assume

A4 the point $\hat{z}$ belongs to $Z_{0}$,

because if A4 failed then we could choose a sufficiently small real number $\mu>0$ with $\bar{x}-\mu f(\bar{x}, \bar{u}) \in Z_{0}$ and study the variational condition

$$
-\mu f(x, u) \in N_{S(u)}(x)
$$

the solution map of which is the same as that of (1).

The definition of $\hat{z}$ and the hypothesis that $-f(\bar{x}, \bar{u}) \in N_{S(\bar{u})}(\bar{x})$ imply that $\bar{x} \in\left(I+N_{S(\bar{u})}\right)^{-1}(\hat{z})$. It then follows from A4 and part (a) of Lemma 3.1 that $\bar{x}=\pi(\bar{u}, \hat{z})$.

The analysis of this section will be based on a condition called the strong coherent orientation condition (SCOC) $[4,17]$, which requires a certain family of matrices to have the same nonzero determinantal sign. To define the SCOC we could use the family $\mathcal{B}$ defined at the beginning of Section 3 , but it suffices to use a subset $\mathcal{B}_{1}$ of $\mathcal{B}$. We define $\mathcal{B}_{1}$ to be the family of sets $K \in \mathcal{B}$ such that (i) there exists $\lambda \in M(\bar{u}, \hat{z})$ with $\operatorname{supp}(\lambda):=\left\{i \in I \cup J: \lambda_{i} \neq 0\right\} \subset K$; (ii) $\operatorname{span}\left\{\nabla_{x} g_{i}(\bar{x}, \bar{u}), i \in K\right\}$ contains $\operatorname{span}\left\{\nabla_{x} g_{i}(\bar{x}, \bar{u}), i \in J\right\}$ as a subspace. This definition is equivalent to definitions of the SCOC family of active index sets in [4, 17], when $J=\emptyset$. In view of part (c) of Lemma 3.1, it is not hard to show via contradiction that there exist neighborhoods $U_{1}$ of $\bar{u}$ in $U_{0}$ and $Z_{1}$ of $\hat{z}$ in $Z_{0}$, with

$$
U_{1}^{\prime}=\left\{u \in U_{1} \mid S(u) \cap X_{0} \neq \emptyset\right\},
$$

such that the following holds:

$\mathbf{A 5}^{\prime}$ For each $(u, z) \in U_{1}^{\prime} \times Z_{1}$ there exists $K \in \mathcal{B}_{1}$ satisfying $\pi(u, z)=x^{K}(u, z), \lambda^{K}(u, z) \in M(u, z)$ and $K \cap I=\operatorname{supp}\left(\lambda^{K}(u, z)\right) \cap I$.

Indeed, in most of this section we only need the following condition, which is slightly weaker than A5'.

A5 For each $(u, z) \in U_{1}^{\prime} \times Z_{1}$ there exists $K \in \mathcal{B}_{1}$ satisfying $\pi(u, z)=x^{K}(u, z)$ and $\lambda^{K}(u, z) \in M(u, z)$.

Condition A5 holds with a family smaller than $\mathcal{B}_{1}$. To see this, define $\mathcal{B}_{2}$ to be the subset of $\mathcal{B}_{1}$ that consists of $K \in \mathcal{B}_{1}$ such that (i) $K$ is a subset of $I(x, u)$ for some $(x, u) \in X_{0} \times U_{0}^{\prime}$ with $x \in S(u)$ and (ii) $\left\{\nabla_{x} g_{i}(x, u), i \in K\right\}$ is a maximal linearly independent subset of $\left\{\nabla_{x} g_{i}(x, u), i \in I(x, u)\right\}$. We show that A5 holds with $\mathcal{B}_{2}$. Let $(u, z) \in U_{1}^{\prime} \times Z_{1}$ and choose $K \in \mathcal{B}_{1}$ such that $\pi(u, z)=x^{K}(u, z)$ and $\lambda^{K}(u, z) \in M(u, z)$, and write $x=\pi(u, z)$ and $\lambda=\lambda^{K}(u, z)$. Because $K \subset I(x, u)$, we may extend $K$ to a set $K_{1} \subset I(x, u)$ such that $\left\{\nabla_{x} g_{i}(x, u), i \in K_{1}\right\}$ is a maximal linearly independent subset of $\left\{\nabla_{x} g_{i}(x, u), i \in I(x, u)\right\}$. The set $K_{1}$ belongs to $\mathcal{B}_{2}$. One can easily verify that $(x, \lambda, u, z)$ satisfies $(15)$ with $K_{1}$ in place of $K$, so $x=x_{K_{1}}(u, z)$ and $\lambda=\lambda_{K_{1}}(u, z)$. This proves that A5 holds with $\mathcal{B}_{2}$ in place of $\mathcal{B}_{1}$. We will use $\mathcal{B}_{1}$ to define the SCOC, but we can replace $\mathcal{B}_{1}$ by $\mathcal{B}_{2}$ wherever it appears in this section except in Corollary 4.3.

In view of $\mathrm{A} 5$, for each $u \in U_{1}^{\prime}$ the function $\pi(u, \cdot)$ is a $P C^{1}$ function on $Z_{1}$ selected from $\left\{x^{K}(u, \cdot), K \in\right.$ $\left.\mathcal{B}_{1}\right\}$. It follows from [33, Corollary 4.1.1 and Proposition 4.1.3] or [17, Lemma 4.2.10] that the B-derivative $d_{z} \pi(u, z)(\cdot)$ for each $(u, z) \in U_{1}^{\prime} \times Z_{1}$ is a continuous function from $\mathbb{R}^{n}$ to $\mathbb{R}^{n}$, with

$$
d_{z} \pi(u, z)(h) \in\left\{\nabla_{z} x^{K}(u, z)(h), K \in \mathcal{B}_{1}\right\}
$$

for each $h \in \mathbb{R}^{n}$. 
Next, define a function $F: U_{1}^{\prime} \times Z_{1} \rightarrow \mathbb{R}^{n}$ by

$$
F(u, z)=f(\pi(u, z), u)+z-\pi(u, z) .
$$

For each $u \in U_{1}^{\prime}$ the map $F(u, \cdot): Z_{1} \rightarrow \mathbb{R}^{n}$ is a localized normal map, induced by the function $f(u, \cdot)$ on $S(u)$. In addition, define for each $K \in \mathcal{B}_{1}$ a $C^{1}$ function $F^{K}: U_{1} \times Z_{1} \rightarrow \mathbb{R}^{n}$ by

$$
F^{K}(u, z)=f\left(x^{K}(u, z), u\right)+z-x^{K}(u, z) .
$$

The fact that $\bar{x}=\pi(\bar{u}, \hat{z})$ and the definition of $\hat{z}$ imply that $F(\bar{u}, \hat{z})=f(\bar{x}, \bar{u})+\hat{z}-\bar{x}=0$. For each $K \in \mathcal{B}_{1}$, the definition of $\mathcal{B}_{1}$ guarantees the existence of $\lambda \in M(\bar{u}, \hat{z})$ with $\operatorname{supp}(\lambda) \subset K$, and one can easily verify that $(\bar{x}, \lambda)$ satisfies $(15)$ under parameter $(\bar{u}, \hat{z})$. Accordingly, we have $\bar{x}=x^{K}(\bar{u}, \hat{z}), \lambda=\lambda^{K}(\bar{u}, \hat{z})$ and $F^{K}(\bar{u}, \hat{z})=0$ for each $K \in \mathcal{B}_{1}$.

By the chain rule of B-differentiability [26, Corollary A.4], for each $u \in U_{1}^{\prime}$ the function $F(u, \cdot)$ is B-differentiable at each $z \in Z_{1}$, with

$$
d_{z} F(u, z)(h)=\nabla_{x} f(x, u) d_{z} \pi(u, z)(h)+h-d_{z} \pi(u, z)(h),
$$

where $d_{z} F(u, z)(h)$ denotes the B-derivative of $F(u, \cdot)$ at $z$ for the direction $h$. This and (47) imply that

$$
d_{z} F(u, z)(h) \in\left\{\nabla_{x} f(x, u) \nabla_{z} x^{K}(u, z)(h)+h-\nabla_{z} x^{K}(u, z)(h), K \in \mathcal{B}_{1}\right\}
$$

for each $(u, z) \in U_{1}^{\prime} \times Z_{1}$ and $h \in \mathbb{R}^{n}$.

For each $(u, z) \in U_{1}^{\prime} \times Z_{1}$ and each $K \in \mathcal{B}_{1}$, if we write $(x, \lambda)=\left(x^{K}(u, z), \lambda^{K}(u, z)\right)$, then the Jacobian matrices of $x^{K}(\cdot, \cdot)$ and $\lambda^{K}(\cdot, \cdot)$ at $(u, z)$ with respect to $z$ are given by (17). Hence, $\nabla_{z} x^{K}(u, z)$ is the left-upper block of the matrix

$$
V^{K}(u, z):=\left[\begin{array}{cc}
I_{n}+\sum_{i \in K} \nabla_{x}^{2} g_{i}(x, u) \lambda_{i} & \nabla_{x} g_{K}(x, u)^{T} \\
-\nabla_{x} g_{K}(x, u) & 0
\end{array}\right]^{-1} .
$$

If we define

$$
\Lambda^{K}(u, z):=\left[\begin{array}{cc}
\nabla_{x} f(x, u)+\sum_{i \in K} \nabla_{x}^{2} g_{i}(x, u) \lambda_{i} & \nabla_{x} g_{K}(x, u)^{T} \\
-\nabla_{x} g_{K}(x, u) & 0
\end{array}\right],
$$

then the product $\Lambda^{K}(u, z) V^{K}(u, z)$ is a block upper triangular matrix, with its left-upper block being

$$
\nabla_{x} f(x, u) \nabla_{z} x^{K}(u, z)+I_{n}-\nabla_{z} x^{K}(u, z),
$$

and its right-lower block being the identity matrix. Because the matrix $I_{n}+\sum_{i \in K} \nabla_{x}^{2} g_{i}(x, u) \lambda_{i}$ is positive definite (see A3 in p.7), we have $\operatorname{det} V^{K}(u, z)>0$. It follows that the matrix displayed in (53), which is the Jacobian matrix of $F^{K}$ with respect to $z$ at $(u, z)$, has the same determinantal sign as $\Lambda^{K}(u, z)$.

The following definition for the SCOC is equivalent to its definitions in $[4,17]$ when $J=\emptyset$.

Definition 4.1 The SCOC holds at $(\bar{x}, \bar{u})$ if for all $K \in \mathcal{B}_{1}$ the matrices $\Lambda^{K}(\bar{u}, \hat{z})$ are of the same nonzero determinantal sign.

By the remark right below (53), if the SCOC holds then the Jacobian matrices of $F^{K}$ for all $K \in \mathcal{B}_{1}$ with respect to $z$ at $(\bar{u}, \hat{z})$ are of the same nonzero determinantal sign. Because $F^{K}(\bar{u}, \hat{z})=0$, by making $U_{1}$ and $Z_{1}$ smaller if necessary we can assume without loss of generality

A6 for each $K \in \mathcal{B}_{1}$ there exists a Lipschitz continuous $C^{1}$ function $z^{K}: U_{1} \rightarrow Z_{1}$ such that $z^{K}(u)$ is the unique solution of $F^{K}(u, \cdot)=0$ in $Z_{1}$.

We can also assume without loss of generality

A7 the matrices $\Lambda^{K}(u, z)$ have a constant nonzero determinant sign, which we denote by $q_{0}$, for all $K \in \mathcal{B}_{1}$ and all $(u, z) \in U_{1}^{\prime} \times Z_{1}$.

The main result of this section is Theorem 4.1. In proving that theorem and its lemmas, we will use some techniques from the degree theory and some results about piecewise affine functions. We will give some basic definitions below. We refer the readers to $[5,13,18]$ for detailed treatments on the degree theory, to $[4,17]$ for summaries of degree-theoretic results that are useful to the analysis here, and to $[4,33]$ for detailed discussions on piecewise affine functions. 
Let $\Omega$ be an open bounded subset of $\mathbb{R}^{n}$, and denote its closure by $\operatorname{cl} \Omega$ and its boundary by bdry $\Omega$. Let $G$ be a continuous function from $\operatorname{cl} \Omega$ to $\mathbb{R}^{n}$. If $a$ is a point of $\mathbb{R}^{n}$ such that $a \notin G($ bdry $\Omega$ ), then the degree of $G$ at $a$ with respect to $\Omega$ is a well defined integer which we denote as $\operatorname{deg}(G \mid a, \Omega)$. The index of $G$ at a point $x_{0} \in \Omega$ is essentially a localized concept of the degree. It is well defined if there exists an open neighborhood $V$ of $x_{0}$ in $\Omega$ such that $G(x) \neq G\left(x_{0}\right)$ for each $x \in \operatorname{cl} V \backslash\left\{x_{0}\right\}$. Let $\mathcal{U}$ be the collection of all open neighborhoods $U$ of $x_{0}$ in $\Omega$ such that $U \subset V$. For each $U \in \mathcal{U}$, the point $G\left(x_{0}\right)$ does not belong to $G\left(\right.$ bdry $U$ ), so $\operatorname{deg}\left(G \mid G\left(x_{0}\right), U\right)$ is well defined. Moreover, by the decomposition-of-domain property and the excision property of degrees [13, Theorem 2.2.1], $\operatorname{deg}\left(G \mid G\left(x_{0}\right), U\right)$ is a constant for all $U \in \mathcal{U}$. We call such a constant the index of $G$ at $x_{0}$ and denote it by $\operatorname{ind}\left(G, x_{0}\right)$.

Next we give the definition of piecewise affine functions. A continuous function $G$ from $\mathbb{R}^{m}$ to $\mathbb{R}^{n}$ is piecewise affine if there exists a finite collection of affine functions $G_{j}: \mathbb{R}^{m} \rightarrow \mathbb{R}^{n}, j=1, \cdots, k$ such that the inclusion $G(x) \in\left\{G_{1}(x), \cdots, G_{k}(x)\right\}$ holds for each $x \in \mathbb{R}^{m}$. The functions $G_{1}, \cdots, G_{k}$ are called selection functions of $G$. Now let $G$ be a piecewise affine function from $\mathbb{R}^{n}$ to $\mathbb{R}^{n}$. If there is a way of choosing its selection functions such that all their Jacobian matrices are of the same nonzero determinantal sign, then we say that $G$ is coherently oriented. Under the SCOC, for each $(u, z) \in U_{1}^{\prime} \times Z_{1}$ the function $d_{z} F(u, z)(\cdot)$ is piecewise affine and coherently oriented, in view of (50), (51), the continuity of $d_{z} \pi(u, z)(\cdot)$ and $\mathrm{A} 7$.

We need the following two lemmas.

Lemma 4.1 Let $G: \mathbb{R}^{n} \rightarrow \mathbb{R}^{n}$ be a global homeomorphism. Then either $\operatorname{ind}(G, x)=1$ for each $x \in \mathbb{R}^{n}$, or ind $(G, x)=-1$ for each $x \in \mathbb{R}^{n}$.

Proof. For each $x \in \mathbb{R}^{n}$, $\operatorname{ind}(G, x)$ is well defined, and is either 1 or -1 by the multiplication theorem [13, Theorem 2.3.1]. Because $\operatorname{ind}(G, \cdot)$ is a continuous function on $\mathbb{R}^{n}$ [13, Theorem 2.1.3], the conclusion of the present lemma follows from the connectivity of $\mathbb{R}^{n}$.

Lemma 4.2 Assume that the $C R C Q$ and $S C O C$ hold at $(\bar{x}, \bar{u})$, and let sets $X_{0}, U_{0}, U_{0}^{\prime}$ and $Z_{0}$ and the function $\pi: U_{0}^{\prime} \times Z_{0} \rightarrow X_{0}$ be determined by Lemma 3.1 and by conditions A1, A2 and A3. Suppose that $A 4$ holds. Choose open neighborhoods $U_{1}$ of $\bar{u}$ in $U_{0}$ and $Z_{1}$ of $\hat{z}$ in $Z_{0}$ and define $U_{1}^{\prime}$ as in (46), such that A5, A6 and A7 hold. Then for each $(u, z) \in U_{1}^{\prime} \times Z_{1}, d_{z} F(u, z)(\cdot)$ is a global Lipschitzian homeomorphism from $\mathbb{R}^{n}$ to $\mathbb{R}^{n}$, and $\operatorname{ind}(F(u, \cdot), z)=q_{0}$.

Proof. Fix $(u, z) \in U_{1}^{\prime} \times Z_{1}$, and let $x=\pi(u, z)$. According to the comments right below the proof of Proposition 3.2, there exists $\lambda \in M(u, z)$ such that the matrix $A:=I_{n}+\sum_{i \in I \cup J} \lambda_{i} \nabla_{x}^{2} g_{i}(x, u)$ is positive definite. It follows from Proposition 3.2 that

$$
d_{z} \pi(u, z) h=\Pi_{K(u, z)}^{A}(h)
$$

for each $h \in \mathbb{R}^{n}$, where $\Pi_{K(u, z)}^{A}(h)$ is defined below the proof of that proposition. Define functions $G$ and $H$ from $\mathbb{R}^{n}$ to $\mathbb{R}^{n}$ by

$$
G(y)=\left[A-I_{n}+\nabla_{x} f(x, u)\right] \Pi_{K(u, z)}(y)+y-\Pi_{K(u, z)}(y)
$$

and

$$
H(y)=\left(I_{n}-A\right) \Pi_{K(u, z)}^{A}(y)+y .
$$

The function $G$ as defined is the normal map induced by the linear transformation $A-I_{n}+\nabla_{x} f(x, u)$ on the polyhedral convex cone $K(u, z)$. By [20, Lemma 8], the function $H$ is a global Lipschitzian homeomorphism from $\mathbb{R}^{n}$ to $\mathbb{R}^{n}$, and its inverse is the normal map induced by the linear transformation $A$ on $K(u, z)$. We have

$$
\begin{aligned}
d_{z} F(u, z) h & =\nabla_{x} f(u, z) \Pi_{K(u, z)}^{A}(h)+h-\Pi_{K(u, z)}^{A}(h) \\
& =G \circ H(h),
\end{aligned}
$$

where the first equality follows from (50) and (54), and the second is from [20, Lemma 9]. Because a normal map induced by a linear map on a polyhedral convex set is a global Lipschitzian homeomorphism if and only if it is coherently oriented (Robinson [27], see also Ralph [22] and Scholtes [34]), $H^{-1}$ is coherently oriented. The function $d_{z} F(u, z)(\cdot)$ is coherently oriented, so $G=d_{z} F(u, z) \circ H^{-1}$ is coherently oriented 
and therefore a global Lipschitzian homeomorphism. Accordingly, $d_{z} F(u, z)(\cdot)$ is a global Lipschitzian homeomorphism.

Because $d_{z} F(u, z)(\cdot)$ is a global homeomorphism, its index is a constant over $\mathbb{R}^{n}$ by Lemma 4.1. On the other hand, because $d_{z} F(u, z)(\cdot)$ is a piecewise linear function, there exists an $n$-dimensional polyhedral convex set, say $C$, on which $d_{z} F(u, z)(\cdot)$ coincides with one of its selection functions [33, Proposition 2.2.3]. The index of $d_{z} F(u, z)(\cdot)$ at each point in int $C$ is equal to the determinantal sign of the Jacobian matrix of that selection function. According to A7, the Jacobian matrices of all selection functions of $d_{z} F(u, z)(\cdot)$ have the same determinantal sign, which is $q_{0}$. Hence, the index of $d_{z} F(u, z)(\cdot)$ is $q_{0}$ over $\mathbb{R}^{n}$. It then follows from [19, Lemma 2] that

$$
\operatorname{ind}(F(u, \cdot), z)=\operatorname{ind}\left(d_{z} F(u, z)(\cdot), 0\right)=q_{0} .
$$

TheOrem 4.1 Assume the hypotheses and notation in Lemma 4.2. There exist neighborhoods $U_{2}$ of $\bar{u}$ in $U_{1}, Z_{2}$ of $\hat{z}$ in $Z_{1}, X_{2}$ of $\bar{x}$ in $X_{0}$, and continuous functions $z$ and $x$ from $U_{2}^{\prime}:=\left\{u \in U_{2} \mid S(u) \cap X_{0} \neq \emptyset\right\}$ to $Z_{2}$ and $X_{2}$ respectively, such that the following hold for each $u \in U_{2}^{\prime}$.

(a) $z(u)$ is the unique solution to the equation $F(u, \cdot)=0$ in $Z_{2}$, and $x(u)$ is the unique solution to (1) in $X_{2}$ under parameter $u$.

(b) The points $x(u)$ and $z(u)$ satisfy

$$
z(u)=x(u)-f(x(u), u) \quad \text { and } \quad x(u)=\pi(u, z(u)) .
$$

(c) There exists $K \in \mathcal{B}_{1}$ such that $z(u)=z^{K}(u), x(u)=x^{K}(u, z(u))$ and $\lambda^{K}(u, z(u)) \in M(u, z(u))$.

Proof. First, we construct the neighborhoods $U_{2}$ and $Z_{2}$ and the function $z$.

Recall that $F(\bar{u}, \hat{z})=0$, and that $\operatorname{ind}(F(\bar{u}, \cdot), \hat{z})=q_{0}$ by Lemma 4.2. There exists an open bounded neighborhood $Z_{2}$ of $\hat{z}$, such that $\operatorname{cl} Z_{2} \subset Z_{1}$, $\operatorname{deg}\left(F(\bar{u}, \cdot), Z_{2}, 0\right)=q_{0}$, and $F(\bar{u}, z) \neq 0$ for each $z \in$ $\operatorname{cl} Z_{2} \backslash\{\hat{z}\}$. We can then find a positive real number $\gamma$ such that

$$
\|F(\bar{u}, z)\| \geq \gamma
$$

for each $z \in \operatorname{bdry} Z_{2}$. Choose another positive real number $b$ such that

$$
\left(\rho+M_{1}+\rho M_{1}\right) b+(1+\rho) M_{2} b^{\frac{1}{2}}<\gamma,
$$

where $M_{1}$ and $M_{2}$ are constants defined in part (b) of Lemma 3.1, and $\rho$ is a constant defined in A2 of p.7. Let $U_{2}=U_{1} \cap(\bar{u}+b B)$, and define $U_{2}^{\prime}$ accordingly.

Next, fix $u \in U_{2}^{\prime}$, and define a homotopy $J: Z_{1} \times[0,1] \rightarrow \mathbb{R}^{n}$ by

$$
J(z, t)=(1-t) F(\bar{u}, z)+t F(u, z) .
$$

For each $z \in \operatorname{bdry} Z_{2}$ and $t \in[0,1]$,

$$
\begin{aligned}
\|J(z, t)\| & =\|(1-t) F(\bar{u}, z)+t F(u, z)\| \\
& \geq\|F(\bar{u}, z)\|-\|F(u, z)-F(\bar{u}, z)\| .
\end{aligned}
$$

We have

$$
\begin{aligned}
\|F(u, z)-F(\bar{u}, z)\| & =\|f(\pi(u, z), u)-\pi(u, z)-f(\pi(\bar{u}, z), \bar{u})+\pi(\bar{u}, z)\| \\
& \leq\|f(\pi(u, z), u)-f(\pi(\bar{u}, z), \bar{u})\|+\|\pi(u, z)-\pi(\bar{u}, z)\| \\
& \leq(1+\rho)\|\pi(u, z)-\pi(\bar{u}, z)\|+\rho\|u-\bar{u}\| \\
& \leq\left(\rho+M_{1}+\rho M_{1}\right)\|u-\bar{u}\|+(1+\rho) M_{2}\|u-\bar{u}\|^{\frac{1}{2}},
\end{aligned}
$$

where the last inequality follows from (19). It then follows from (56) and the fact that $u \in \bar{u}+b B$ that

$$
\begin{aligned}
\|J(z, t)\| & \geq \gamma-\left(\rho+M_{1}+\rho M_{1}\right) b-(1+\rho) M_{2} b^{\frac{1}{2}} \\
& >0 .
\end{aligned}
$$


Hence, $J(z, t) \neq 0$ for each $z \in$ bdry $Z_{2}$ and $t \in[0,1]$. The homotopy invariance theorem [13, Theorem 2.1.2] then yields

$$
\operatorname{deg}\left(F(u, \cdot), Z_{2}, 0\right)=\operatorname{deg}\left(F(\bar{u}, \cdot), Z_{2}, 0\right)=q_{0},
$$

so the equation $F(u, \cdot)=0$ has at least one solution in $Z_{2}$.

To prove solution uniqueness, note that

$$
F(u, z)=J(z, 1) \neq 0
$$

for each $z \in \operatorname{bdry} Z_{2}$, and recall that $\operatorname{ind}(F(u, \cdot), z)=q_{0}$ at each $z \in Z_{2}$ by Lemma 4.2. Suppose for the purpose of contradiction that the equation $F(u, \cdot)=0$ has infinitely many solutions in $Z_{2}$. Each cluster point of such solutions satisfies $F(u, \cdot)=0$ and belongs to $Z_{2}$. The index of $F(u, \cdot)$ at such a cluster point is not well defined, a contradiction. Hence, the equation $F(u, \cdot)=0$ has only finitely many solutions in $Z_{2}$. The fact that it has a unique solution in $Z_{2}$ follows from the equality (57) and the decomposition-of-domain property of degrees.

Denote the unique solution of $F(u, \cdot)=0$ in $Z_{2}$ by $z(u)$ for each $u \in U_{2}^{\prime}$. We prove that $z(\cdot)$ is continuous on $U_{2}^{\prime}$. Let $u$ be a point in $U_{2}^{\prime}$, and let $\left\{u_{k}\right\}$ be a sequence in $U_{2}^{\prime}$ converging to $u$. Let $z$ be a cluster point of the sequence $\left\{z\left(u_{k}\right)\right\}$. The continuity of $F$ implies that $F(u, z)=0$, and it follows from (58) that $z \in Z_{2}$. As a result, $z=z(u)$. This proves that the sequence $\left\{z\left(u_{k}\right)\right\}$ converges to $z(u)$, and thereby proves continuity.

Next, define a continuous function $x(\cdot)$ from $U_{2}^{\prime}$ to $X_{0}$ by

$$
x(u)=\pi(u, z(u)) .
$$

By reducing the sizes of $U_{2}$ and $U_{2}^{\prime}$, we may find a neighborhood $X_{2}$ of $\bar{x}$ in $X_{0}$, such that $x-f(x, u) \in Z_{2}$ for each $(x, u) \in X_{2} \times U_{2}^{\prime}$ and $x(u) \in X_{2}$ for each $u \in U_{2}^{\prime}$. For each such $u$, we have

$$
0=F(u, z(u))=f(x(u), u)+z(u)-x(u) .
$$

The two equations in (55) follow from (59) and (60). Equation (59) and part (a) of Lemma 3.1 imply that

$$
z(u)-x(u) \in N_{S(u)}(x(u)) .
$$

The latter inclusion and (60) implies that $x(u)$ satisfies (1).

To show that for each $u \in U_{2}^{\prime}$ the point $x(u)$ is the unique solution to (1) in $X_{2}$, suppose that $x^{\prime}$ belongs to $X_{2}$ and satisfies $0 \in f\left(x^{\prime}, u\right)+N_{S(u)}\left(x^{\prime}\right)$. Define $z^{\prime}$ by

$$
z^{\prime}=x^{\prime}-f\left(x^{\prime}, u\right)
$$

This definition implies that $z^{\prime}-x^{\prime} \in N_{S(u)}\left(x^{\prime}\right)$ and that $z^{\prime} \in Z_{2}$. By part (a) of Lemma 3.1 we have $x^{\prime}=\pi\left(u, z^{\prime}\right)$, which together with (61) implies that $F\left(u, z^{\prime}\right)=0$. It follows that $z^{\prime}=z(u)$ and $x^{\prime}=x(u)$. This proves that $x(u)$ is the unique solution to (1) in $X_{2}$.

It remains to prove part (c). According to A5, there exists some $K \in \mathcal{B}_{1}$ such that $x(u)=\pi(u, z(u))=$ $x^{K}(u, z(u))$ and $\lambda^{K}(u, z(u)) \in M(u, z(u))$. It follows that $0=F(u, z(u))=F^{K}(u, z(u))$. This implies that $z(u)=z^{K}(u)$ in view of the definition of $z^{K}$ in A6.

Functions $z(\cdot)$ and $x(\cdot)$ given in Theorem 4.1 are continuous and satisfy $z(u) \in\left\{z^{K}(u), K \in \mathcal{B}_{1}\right\}$ and $x(u) \in\left\{x^{K}\left(u, z^{K}(u)\right), K \in \mathcal{B}_{1}\right\}$ respectively. By their definitions, $z^{K}$ and $x^{K}$ are Lipschitz continuous functions, so it follows from [33, Proposition 4.1.2] that $z(\cdot)$ and $x(\cdot)$ are Lipschitz continuous as long as their domain $U_{2}^{\prime}$ is convex.

The set $U_{2}^{\prime}$ is a neighborhood of $\bar{u}$ in $\left\{u \in \bar{U} \mid S(u) \cap X_{0} \neq \emptyset\right\}$. In general, the latter set is not necessarily convex, and one cannot expect $U_{2}^{\prime}$ to be convex. In the following, we discuss two special cases in which one can find a convex neighborhood $U_{3}^{\prime}$ of $\bar{u}$ in $U_{2}^{\prime}$. Accordingly, the restrictions of $z$ and $x$ on $U_{3}^{\prime}$ are Lipschitz continuous.

The first case is when the MFCQ holds at $(\bar{x}, \bar{u})$ in addition to the CRCQ and SCOC.

DEFINITION 4.2 The MFCQ holds at $(\bar{x}, \bar{u})$ if

(a) there exists a vector $w \in \mathbb{R}^{n}$ such that

$$
\begin{aligned}
\left\langle\nabla_{x} g_{i}(\bar{x}, \bar{u}), w\right\rangle & <0, \quad i \in I(\bar{x}, \bar{u}) \cap I, \\
& =0, \quad i \in J
\end{aligned}
$$


(b) the family $\left\{\nabla_{x} g_{i}(\bar{x}, \bar{u}), \quad i \in J\right\}$ has full rank $|J|$.

COROLlary 4.1 Assume the hypotheses of Theorem 4.1, and assume additionally that the MFCQ holds at $(\bar{x}, \bar{u})$. Determine sets $U_{2}, U_{2}^{\prime}, Z_{2}, X_{2}$ and functions $z: U_{2}^{\prime} \rightarrow Z_{2}$ and $x: U_{2}^{\prime} \rightarrow X_{2}$ as in that theorem. There exists a neighborhood $U_{3}^{\prime}$ of $\bar{u}$ in $U_{2}^{\prime}$, such that $U_{3}^{\prime}$ is an open and convex subset of $\mathbb{R}^{m}$ and that the restrictions of $z$ and $x$ to $U_{3}^{\prime}$ are Lipschitz continuous $P C^{1}$ functions.

Proof. Under the MFCQ, there exists an open neighborhood $U$ of $\bar{u}$ in $\mathbb{R}^{m}$ such that $S(u) \cap X_{0} \neq \emptyset$ for each $u \in U$, see, e.g., [23, Theorem 1 and 3]. Because $U_{2}^{\prime}=\left\{u \in U_{2} \mid S(u) \cap X_{0} \neq \emptyset\right\}$ by its definition, we have $U_{2}^{\prime} \cap U=U_{2} \cap U$, which is a neighborhood of $\bar{u}$ in $\mathbb{R}^{m}$. We can then find an open and convex set $U_{3}^{\prime}$ in $\mathbb{R}^{m}$, with $\bar{u} \in U_{3}^{\prime} \subset U_{2}^{\prime} \cap U$. The restrictions of $z$ and $x$ to $U_{3}^{\prime}$ are Lipschitz continuous $P C^{1}$ functions.

Corollary 4.1 extends an earlier result on variational inequalities under the MFCQ, CRCQ and SCOC, see [17, Theorem 4.2.16] and [4, Theorem 5.4.12], which assumes additionally that $J=\emptyset$ and that $g_{i}$ are convex in $x$ for each $i \in I$.

The second case is when the functions $g_{i}$ are affine. Applying Theorem 4.1 to this case resulted in the following corollary, which is equivalent to part of [16, Theorem 5.2] if we replace $\mathcal{B}_{1}$ by $\mathcal{B}_{2}$ in the definition of the SCOC.

Corollary 4.2 Assume the hypotheses of Theorem 4.1, and assume additionally that for each $i \in I \cup J$ the function $g_{i}$ is affine. Determine sets $U_{2}, U_{2}^{\prime}, Z_{2}, X_{2}$ and functions $z: U_{2}^{\prime} \rightarrow Z_{2}$ and $x: U_{2}^{\prime} \rightarrow X_{2}$ as in that theorem. There exists a convex neighborhood $U_{3}^{\prime}$ of $\bar{u}$ in $U_{2}^{\prime}$, such that the restrictions of $z$ and $x$ to $U_{3}^{\prime}$ are Lipschitz continuous functions.

Proof. Define a multifunction $G$ from $\mathbb{R}^{m}$ to $\mathbb{R}^{n}$ by $G(u)=\left\{x \in \mathbb{R}^{n} \mid g_{i}(x, u) \leq 0, i \in I, \quad g_{i}(x, u)=\right.$ $0, i \in J\}$. Because the functions $g_{i}$ are affine, the set $\operatorname{dom} G:=\left\{u \in \mathbb{R}^{m} \mid G(u) \neq \emptyset\right\}$ is a polyhedral convex set, and $G$ is a Lipschitz continuous multifunction on dom $G$. Hence, there exists a convex neighborhood $U$ of $\bar{u}$ in $\operatorname{dom} G$ such that $G(u) \cap X_{0} \neq \emptyset$ for each $u \in U$. Because $U_{2}^{\prime}=\left\{u \in U_{2}\right.$ | $\left.S(u) \cap X_{0} \neq \emptyset\right\}$ by its definition, we have $U_{2}^{\prime} \cap U=U_{2} \cap U$. Let $U_{3}^{\prime}$ be a convex neighborhood of $\bar{u}$ in $U_{2}^{\prime} \cap U$; the restrictions of $z$ and $x$ to $U_{3}^{\prime}$ are Lipschitz continuous functions.

Finally, we apply Theorem 4.1 to a parametric nonlinear program

$$
\begin{aligned}
& \min \varphi(x, u) \\
& \text { s.t. } x \in S(u)
\end{aligned}
$$

where $\varphi$ is a $C^{2}$ function from the open set $\bar{X} \times \bar{U}$ to $\mathbb{R}$, and $S(u)$ is as defined in (2). The corollary below uses a second order condition, which implies the SCOC to hold.

Corollary 4.3 Define $f(x, u):=\nabla_{x} \varphi(x, u)$, and suppose that $(\bar{x}, \bar{u}) \in \bar{X} \times \bar{U}$ satisfies (1) and the $C R C Q$. Let sets $X_{0}, U_{0}, U_{0}^{\prime}$ and $Z_{0}$ and the function $\pi: U_{0}^{\prime} \times Z_{0} \rightarrow X_{0}$ be determined by Lemma 3.1 and by conditions A1, A2 and A3. Let $\hat{z}=\bar{x}-f(\bar{x}, \bar{u})$, and suppose that A4 holds. Suppose that for each $K \in \mathcal{B}_{1}$ and each $v \in \mathbb{R}^{n} \backslash\{0\}$ with

$$
\nabla_{x} g_{K}(\bar{x}, \bar{u}) v=0,
$$

one has $\left\langle v, \nabla_{x} f(\bar{x}, \bar{u}) v+\sum_{i \in K} \nabla_{x}^{2} g_{i}(\bar{x}, \bar{u}) \lambda_{i}^{K}(\bar{u}, \hat{z}) v\right\rangle>0$. There exist neighborhoods $U_{2}$ of $\bar{u}$ in $U_{0}, Z_{2}$ of $\hat{z}$ in $Z_{0}, X_{2}$ of $\bar{x}$ in $X_{0}$, and continuous functions $z$ and $x$ from $U_{2}^{\prime}:=\left\{u \in U_{2} \mid S(u) \cap X_{0} \neq \emptyset\right\}$ to $Z_{2}$ and $X_{2}$ respectively, such that the following hold for each $u \in U_{2}^{\prime}$.

(a) $z(u)$ is the unique solution to the equation $F(u, \cdot)=0$ in $Z_{2}$, and $x(u)$ is the unique local minimum of (62) in $X_{2}$.

(b) The points $x(u)$ and $z(u)$ satisfy

$$
z(u)=x(u)-f(x(u), u) \quad \text { and } \quad x(u)=\pi(u, z(u)) .
$$

(c) There exists $K \in \mathcal{B}_{1}$ such that $z(u)=z^{K}(u), x(u)=x^{K}(u, z(u)), \lambda^{K}(u, z(u)) \in M(u, z(u))$ and $K \cap I=\operatorname{supp} \lambda^{K}(u, z(u)) \cap I$. 
Proof. We start with a notational definition. For each $K \in \mathcal{B}_{1}, u \in U_{0}$ and $z \in Z_{0}$, write $(x, \lambda)=\left(x^{K}(u, z), \lambda^{K}(u, z)\right)$ to define

$$
\Gamma^{K}(u, z)=\nabla_{x} f(x, u)+\sum_{i \in K} \nabla_{x}^{2} g_{i}(x, u) \lambda_{i} .
$$

Now, for each $K \in \mathcal{B}_{1}$, let $E$ be a matrix whose columns form a basis of the null space of $\nabla_{x} g_{K}(\bar{x}, \bar{u})$, and recall that $\bar{x}=x^{K}(\bar{u}, \hat{z})$. The second-order hypothesis implies that the matrix $E^{T} \Gamma^{K}(\bar{u}, \hat{z}) E$ is positive definite. Because $\nabla_{x} g_{K}(\bar{x}, \bar{u})$ is of full row rank, we have

$$
\operatorname{det} \Lambda^{K}(\bar{u}, \hat{z})=\operatorname{det}\left[\begin{array}{cc}
\Gamma^{K}(\bar{u}, \hat{z}) & \nabla_{x} g_{K}(\bar{x}, \bar{u})^{T} \\
-\nabla_{x} g_{K}(\bar{x}, \bar{u}) & 0
\end{array}\right]=\operatorname{det}\left(E^{T} \Gamma^{K}(\bar{u}, \hat{z}) E\right)>0,
$$

by an application of [33, Lemma 5.2.1]; see also [10, Theorem 3.5]. This shows that the SCOC holds at $(\bar{x}, \bar{u})$. We can then choose open neighborhoods $U_{1}$ of $\bar{u}$ in $U_{0}$ and $Z_{1}$ of $\hat{z}$ in $Z_{0}$ and define $U_{1}^{\prime}$ as in (46), such that $\mathrm{A} 5^{\prime}, \mathrm{A} 6$ and $\mathrm{A} 7$ hold. By reducing the sizes of $U_{1}$ and $Z_{1}$ if necessary, we may assume

$$
v \neq 0, \nabla_{x} g_{K}\left(x^{K}(u, z), u\right) v=0 \quad \Rightarrow \quad\left\langle v, \Gamma^{K}(u, z) v\right\rangle>0
$$

for each $K \in \mathcal{B}_{1}, u \in U_{1}^{\prime}$ and $z \in Z_{1}$.

Next, apply Theorem 4.1 to obtain neighborhoods $U_{2}$ of $\bar{u}$ in $U_{1}, Z_{2}$ of $\hat{z}$ in $Z_{1}, X_{2}$ of $\bar{x}$ in $X_{0}$, and continuous functions $z$ and $x$ from $U_{2}^{\prime}:=\left\{u \in U_{2} \mid S(u) \cap X_{0} \neq \emptyset\right\}$ to $Z_{2}$ and $X_{2}$ respectively, such that parts (a), (b) and (c) of Theorem 4.1 hold for each $u \in U_{2}^{\prime}$. The fact that A5' holds ensures part (c) of the present theorem to hold.

It remains to show that $x(u)$ is the unique local minimum of (62) in $X_{2}$ for each $u \in U_{2}^{\prime}$. Choose $u \in U_{2}^{\prime}$; we just showed that there exists $K \in \mathcal{B}_{1}$ such that $z(u)=z^{K}(u), x(u)=x^{K}(u, z(u)), \lambda^{K}(u, z(u)) \in$ $M(u, z(u))$ and $K \cap I=\operatorname{supp}\left(\lambda^{K}(u, z(u)) \cap I\right.$. In view of (64), $x(u)$ satisfies the well known second order sufficient condition, so it is a strict local minimum of (62). Because the CRCQ holds at $(x, u)$ for any $x \in X_{2}$ (see A1), any local minimum in $X_{2}$ must satisfy (1). Because $x(u)$ is the unique solution to (1) in $X_{2}$, it is the unique local minimum in $X_{2}$.

In the above proof we used condition A $5^{\prime}$ instead of $\mathrm{A} 5$. Because $\mathrm{A} 5^{\prime}$ does not hold with $\mathcal{B}_{2}$, we cannot replace $\mathcal{B}_{1}$ by $\mathcal{B}_{2}$ in the statement of the above theorem. This is the only place in this section where we cannot replace $\mathcal{B}_{1}$ by $\mathcal{B}_{2}$.

The second order condition in the statement of Corollary 4.3 is closely related to Condition 7.3 in [10], called the general strong second order sufficient condition (GSSOSC) in [11, 12]. In the notation here, that condition requires that for each $\lambda \in M(\bar{u}, \hat{z})$ and each $v \in \mathbb{R}^{n} \backslash\{0\}$ with

$$
\nabla_{x} g_{i}(\bar{x}, \bar{u}) v=0, \quad i \in(\operatorname{supp}(\lambda) \cap I) \cup J,
$$

one has $\left\langle v, \nabla_{x} f(\bar{x}, \bar{u}) v+\sum_{i \in I \cup J} \nabla_{x}^{2} g_{i}(\bar{x}, \bar{u}) \lambda_{i} v\right\rangle>0$. Recall that for each $K \in \mathcal{B}_{1}$, the point $\lambda^{K}(\bar{u}, \hat{z})$ belongs to $M(\bar{u}, \hat{z})$. One can readily verify that the GSSOSC implies the second order condition in Corollary 4.3 .

Acknowledgments. This material is based upon research supported by the National Science Foundation under Grant DMS-0807893. The author thanks Professors Stephen M. Robinson, Paul Tseng and two anonymous referees for comments that have greatly improved this paper.

\section{References}

[1] J. Fréderic Bonnans and Alxander Shapiro. Perturbation Analysis of Optimization Problems. Springer Series in Operations Research. Springer-Verlag, New York, 2000.

[2] S. Dempe. Directional differentiability of optimal solutions under Slater's condition. Mathematical Programming, 59:49-69, 1993.

[3] A. L. Dontchev and R. T. Rockafellar. Implicit Functions and Solution Mappings: A View from Variational Analysis. Springer Monographs in Mathematics. Springer, 2009.

[4] Francisco Facchinei and Jong-Shi Pang. Finite-Dimensional Variational Inequalities and Complementarity Problems. Springer Series in Operations Research. Springer-Verlag, New York, 2003. Published in two volumes, paginated continuously. 
[5] Irene Fonseca and Wilfrid Gangbo. Degree Theory in Analysis and Applications, volume 2 of Oxford Lecture Series in Mathematics and its Applications. Oxford University Press, Oxford, 1995.

[6] J. Guddat, F. Guerra Vasquez, and H. Th. Jongen. Parametric Optimization: Singularities, Pathfollowing and Jumps. B. G. Teubner and John Wiley \& Sons, Stuttgart and Chichester, 1990.

[7] A. Haraux. How to differentiate the projection on a convex set in hilbert space. Some applications to variational inequalities. Journal of the Mathematical Society of Japan, 29:615-631, 1977.

[8] R. Janin. Directional derivative of the marginal function in nonlinear programming. Mathematical Programming Studies, 21:110-126, 1984.

[9] Diethard Klatte and Bernd Kummer. Nonsmooth Equations in Optimization: Regularity, Calculus, Methods and Applications. Kluwer Academic Publishers, Dordrecht, 2002.

[10] Masakazu Kojima. Strongly stable stationary solutions in nonlinear programs. In Stephen M. Robinson, editor, Analysis and Computation of Fixed Points, pages 93-138, New York, 1980. Academic Press.

[11] Jerzy Kyparisis. Sensitivity analysis for nonlinear programs and variational inequalities with nonunique multipliers. Mathematics of Operations Research, 15:286-298, 1990.

[12] Jiming Liu. Sensitivity analysis in nonlinear programs and variational inequalities via continuous selections. SIAM Journal on Control and Optimization, 33:1040-1060, 1995.

[13] N. G. LLoyd. Degree Theory. Cambridge University Press, Cambridge, 1978.

[14] Shu Lu. Implications of the constant rank constraint qualification. Mathematical Programming, 2009. Published online at DOI 10.1007/s10107-009-0288-3.

[15] Shu Lu and Stephen M. Robinson. Normal fans of polyhedral convex sets: Structures and connections. Set-valued Analysis, 16:281-305, 2008.

[16] Shu Lu and Stephen M. Robinson. Variational inequalities over perturbed polyhedral convex sets. Mathematics of Operations Research, 33:689-711, 2008.

[17] Z.-Q. Luo, J.-S. Pang, and D. Ralph. Mathematical Programs with Equilibrium Constraints. Cambridge University Press, 1996.

[18] J. M. Ortega and W. C. Rheinboldt. Iterative Solution of Nonlinear Equations in Several Variables. Academic Press, New York, 1970.

[19] Jong-Shi Pang. A degree-theoretic approach to parametric nonsmooth equations with multivalued solution sets. Mathematical Programming, 62:359-383, 1993.

[20] Jong-Shi Pang and Daniel Ralph. Piecewise smoothness, local invertibility, and parametric analysis of normal maps. Mathematics of Operations Research, 21:401-426, 1996.

[21] D. Ralph and S. Dempe. Directional derivatives of the solution of a parametric nonlinear program. Mathematical Programming, 70:159-172, 1995.

[22] Daniel Ralph. On branching numbers of normal manifolds. Nonlinear Analysis: Theory, Methods, and Applications, 22:1041-1050, 1994.

[23] Stephen M. Robinson. Stability theory for systems of inequalities, Part II: Differentiable nonlinear systems. SIAM Journal on Numerical Analysis, 13:497-513, 1976.

[24] Stephen M. Robinson. Generalized equations and their solutions, Part II: Applications to nonlinear programming. Mathematical Programming Studies, 19:200-221, 1982.

[25] Stephen M. Robinson. Local structure of feasible sets in nonlinear programming, Part II: Nondegeneracy. Mathematical Programming Studies, 22:217-230, 1984.

[26] Stephen M. Robinson. Local structure of feasible sets in nonlinear programming, Part III: Stability and sensitivity. Mathematical Programming Studies, 30:45-66, 1987.

[27] Stephen M. Robinson. Normal maps induced by linear transformations. Mathematics of Operations Research, 17:691-714, 1992.

[28] Stephen M. Robinson. Constraint nondegeneracy in variational analysis. Mathematics of Operations Research, 28:201-232, 2003.

[29] Stephen M. Robinson. Variational conditions with smooth constraints: structure and analysis. Mathematical Programming Series B, 97:245-265, 2003. 
[30] Stephen M. Robinson. Localized normal maps and the stability of variational conditions. Set-Valued Analysis, 12:259-274, 2004. Errata, Set-Valued Analysis 14 (2006) 207.

[31] R. Tyrrell Rockafellar. Convex Analysis. Princeton University Press, Princeton, NJ, 1970.

[32] R. Tyrrell Rockafellar and Roger J-B Wets. Variational Analysis. Springer-Verlag, Berlin, 1998.

[33] Stefan Scholtes. Introduction to Piecewise Differentiable Equations. Habilitationsschrift, Institut für Statistik und mathematische Wirtschaftstheorie, Universität Fridericiana Karlsruhe, Karlsruhe, Germany, 1994.

[34] Stefan Scholtes. A proof of the branching number bound for normal manifolds. Linear Algebra and its Applications, 246:83-95, 1996. 